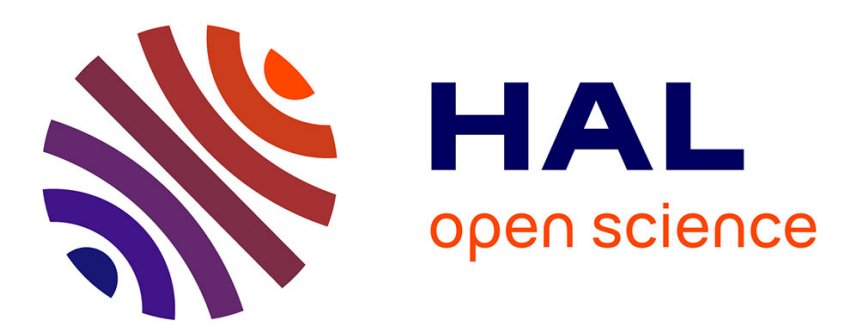

\title{
Theoretical and empirical scale dependency of Z-R relationships: Evidence, impacts, and correction
}

Sébastien Verrier, Laurent Barthès, Cécile Mallet

\section{To cite this version:}

Sébastien Verrier, Laurent Barthès, Cécile Mallet. Theoretical and empirical scale dependency of Z-R relationships: Evidence, impacts, and correction. Journal of Geophysical Research: Atmospheres, 2013, 118 (14), pp.7435-7449. 10.1002/jgrd.50557 . hal-00851593

\section{HAL Id: hal-00851593 https://hal.science/hal-00851593}

Submitted on 31 Aug 2020

HAL is a multi-disciplinary open access archive for the deposit and dissemination of scientific research documents, whether they are published or not. The documents may come from teaching and research institutions in France or abroad, or from public or private research centers.
L'archive ouverte pluridisciplinaire HAL, est destinée au dépôt et à la diffusion de documents scientifiques de niveau recherche, publiés ou non, émanant des établissements d'enseignement et de recherche français ou étrangers, des laboratoires publics ou privés. 


\title{
Theoretical and empirical scale dependency of Z-R relationships: Evidence, impacts, and correction
}

\author{
Sébastien Verrier, ${ }^{1,2,3}$ Laurent Barthès, ${ }^{1}$ and Cécile Mallet ${ }^{1}$ \\ Received 27 September 2012; revised 4 June 2013; accepted 4 June 2013; published 16 July 2013.
}

[1] Estimation of rainfall intensities from radar measurements relies to a large extent on power-laws relationships between rain rates $R$ and radar reflectivities $Z$, i.e., $Z=a^{*} R^{\wedge} b$. These relationships are generally applied unawarely of the scale, which is questionable since the nonlinearity of these relations could lead to undesirable discrepancies when combined with scale aggregation. Since the parameters $(a, b)$ are expectedly related with drop size distribution (DSD) properties, they are often derived at disdrometer scale, not at radar scale, which could lead to errors at the latter. We propose to investigate the statistical behavior of $Z-R$ relationships across scales both on theoretical and empirical sides. Theoretically, it is shown that claimed multifractal properties of rainfall processes could constrain the parameters $(a, b)$ such that the exponent $b$ would be scale independent but the prefactor $a$ would be growing as a (slow) power law of time or space scale. In the empirical part (which may be read independently of theoretical considerations), high-resolution disdrometer (Dual-Beam Spectropluviometer) data of rain rates and reflectivity factors are considered at various integration times comprised in the range $15 \mathrm{~s}-64 \mathrm{~min}$. A variety of regression techniques is applied on $Z-R$ scatterplots at all these time scales, establishing empirical evidence of a behavior coherent with theoretical considerations: $a$ grows as a 0.1 power law of scale while $b$ decreases more slightly. The properties of $a$ are suggested to be closely linked to inhomogeneities in the DSDs since extensions of $Z-R$ relationships involving (here, strongly nonconstant) normalization parameters of the DSDs seem to be more robust across scales. The scale dependence of simple $Z=a^{*} R^{\wedge} b$ relationships is advocated to be a possible source of overestimation of rainfall intensities or accumulations. Several ways for correcting such scaling biases (which can reach $>15-20 \%$ in terms of relative error) are suggested. Such corrections could be useful in some practical cases where $Z-R$ scale biases are significant, which is especially expected for convective rainfall.

Citation: Verrier, S., L. Barthès, and C. Mallet (2013), Theoretical and empirical scale dependency of Z-R relationships: Evidence, impacts, and correction, J. Geophys. Res. Atmos., 118, 7435-7449, doi:10.1002/jgrd.50557.

\section{Introduction}

[2] Radar-rainfall estimates are generally computed from radar measurements using (semi-) theoretical relationships between the reflectivity $Z$ and the rain rate $R$. The most classical relationships consist of power laws of the form:

$$
Z=a R^{b}
$$

where $a$ and $b$ are unknown constants. These constants are strongly dependent on the shape of the DSD. For instance, in the case of a Gamma DSD with parameters $\left(\mathrm{N}_{0}, \mu, \Lambda\right)$,

\footnotetext{
${ }^{1}$ Université Versailles Saint Quentin, LATMOS/IPSL (UMR 8190) INSU/CNRS, Guyancourt, France.

${ }^{2}$ Laboratoire d'Océanographie et du Climat: Expérimentations et Approches Numériques (LOCEAN/UPMC/IPSL), Paris, France.

${ }^{3}$ Centre National d'Etudes Spatiales (CNES), Paris, France.

Corresponding author: S. Verrier, Université de Versailles Saint-Quentin-enYvelines, CNRS/INSU, LATMOS - Laboratoire Atmosphères, Milieux, Observations Spatiales, Guyancourt FR-78280, France. (verrier@latmos.ipsl.fr)

(C)2013. American Geophysical Union. All Rights Reserved. 2169-897X/13/10.1002/jgrd.50557
}

equation (1) may be retrieved theoretically with $b$ depending on $\mu$ and $a$ on the couple $\left(\mathrm{N}_{0}, \mu\right)$. Less restrictive hypotheses on the mathematical form of the DSD are also compatible with equation (1): a more general case corresponds to the "scaling" expression of DSDs involving normalization by a single DSD moment [e.g., Sempere Torres et al., 1994; Uijlenhoet, 2001].

[3] Also, it has been shown that more accurate "double" power laws could be found by normalizing with two reference DSD moments [Testud et al., 2001; Lee et al., 2004].

[4] However, the present study will be mainly devoted to the classical case expressed by equation (1), except when explicitly stated. In particular, (double) normalized power laws in the form expressed by Testud et al. [2001] will be considered more closely in section 6 .

[5] However, this method for estimating rainfall rates remains subject to many uncertainties. Numerous empirical studies have been carried out to estimate the parameters $a$ and $b$ which were found to vary in a large range of values 30-1000 and 0.8-2 [e.g., Battan, 1973; Smith and Krajewski, 1993; see $Y u, 2012$ for a recent review]. Various physical and statistical phenomena are expected to contribute to this 
variability. The parameters $a$ and $b$ are known to depend on the rainfall type (i.e., convective vs. stratiform), but also on the more subtle physical processes involved in rainfall [Rosenfeld and Ulbrich, 2003] that impact the DSD shape, such as coalescence, breakup, or evaporation.

[6] Complementary to these physical factors, it has also been demonstrated that other methodological and statistical factors affect $Z-R$ relationships. Especially, $Z-R$ relationship parameters are highly dependent on methodological aspects such as the type of regression [Campos and Zawadzki, 2000]: for instance, linear regression on the $\operatorname{logs}$ of $Z$ and $R$ and direct nonlinear regressions on $Z$ and $R$ will not provide the same parameters. Even with a given methodology, the result will be sensitive to the choice of the "explicative" variable (that is, $Z$ vs. $R$ and $R$ vs. $Z$ regression will provide different parameters). On the other hand, observational errors and uncertainties will affect the relationships in a significant way [Ciach and Krajewski, 1999; Morin et al., 2003].

[7] Furthermore, it should be emphasized that while the DSD should be robustly defined over large spatial and temporal domains of integration, it may be expected to be largely variable in the interior of such domains [Uijlenhoet et al., 2003; Lee and Zawadzki, 2005; Berne and Uijlenhoet, 2005; Chapon et al., 2007]. Therefore, the parameters of the DSD should be sensitive to aggregation over domains of different volumes and durations, leading to scale-dependent $Z-R$ relations. It is in fact not surprising that $Z-R$ parameters may vary with scale, since the existence of a unique, nonlinear, power law like equation (1) is incompatible with the physical scale-by-scale conservation of both $R$ and $Z$ (while $b$-th and $1 / b$-th order moments of inhomogeneous $R$ and $Z$ have no reason to be conserved with scale). Moreover, $R$ and $Z$ are known from a long time to follow scaling/multifractal properties that describe statistically their variability [Tessier et al., 1993]. These properties reveal to be very convenient for understanding $Z-R$ laws in a scale-dependent framework.

[8] The scale dependency of $Z-R$ relationships has also been documented on more empirical grounds by some authors. Morin et al. [2003] have shown empirically the existence of a scale dependency of $Z-R$ law parameters based on the study of collocated radar and rain gauge data aggregated at different scales $(1-5 \mathrm{~km}, 5-120 \mathrm{~min})$. These authors found a quick increase of the parameter $a$ with scale as well as a moderate decrease of the parameter $b$. They attributed a significant part of the change in parameters to the averaging of observational errors with pixel aggregation. However, they cannot distinguish the purely statistical contribution of rain inhomogeneities since their study involved systematic comparison of radar data and rain gauges which adds instrumental and methodological errors. Then, Steiner and Smith [2004] used Joss-Waldvogel [1967] disdrometer data aggregated at some time scales to support the existence of a $Z-R$ relationship scale dependency as a significant source of errors in radar-rainfall rates. There results were qualitatively consistent with Morin et al.'s study. Lovejoy et al. [2008] studied series of reflectivities measured by the spaceborne radar of TRMM and found the existence of wide range scaling properties of the reflectivities, indicating extreme variability. In this context, they showed that the use of a single, scale independent, $Z-R$ relationship could lead to important errors in the estimation of the mean rain rate (up to a factor 5 over the full range of spatial scales of TRMM data!).
More recently, Jaffrain and Berne [2012] have used the data from a network of 16 PARSIVEL optical disdrometer. Their disdrometers were distributed over a single radar pixel. They estimated $a$ and $b$ from the scatterplots of the point values of $R$ and $Z$ (here the reflectivity factor), and also from the scatterplots of the 16 stations averages (which were supposed to provide a better estimate of radar pixel size variables). They found a significant increase of $a$ with space "scale" and a very low increase in $b$. From this methodology, Jaffrain and Berne demonstrated that the use of small-scale-derived $a$ and $b$ parameters could lead to a biased rainfall estimate at pixel size (generally an overestimation up to $15 \%$ ).

[9] The goal of the present paper is to investigate the scale dependency of $Z-R$ relationships both theoretically and empirically. Theoretically, we start from a stochastic description of time/space rainfall processes based on the theory of fractals and multiplicative cascades to assess the scale behavior of $Z-R$ relationships. The demonstration reuses some previous mathematical results established in universal multifractal theory [Tessier et al., 1993; Lovejoy et al., 2008]. The theoretical framework predicts a constant parameter $b$ while the parameter $a$ should grow as a power law of scale. The other sections of the paper consist of an empirical study to check whether the (at least qualitative) predictions of the theory are valid on data. More precisely, the empirical work consists in the systematic study of the measurements of a specific disdrometer, the Dual-Beam Spectropluviometer (DBS). From this instrument, two-year high-resolution (15 s) time series of rain rate and reflectivity factors are available. These series are degraded at various coarser resolutions leading to an estimation of the parameters $a$ and $b$ at all these scales. Note that the empirical work may be read mainly independently of the theoretical sections (i.e., sections 2 and 3, see below) and does not involve multifractal theory. Readers that are not familiar with the multifractal theory may skip sections 2 at a first time if necessary (except equation (20)).

[10] The methodology followed in this study differs from the previous ones from a few aspects. First, a single instrument is used, which avoids problems of comparison of different instruments. The range of scales considered here is larger than that of Steiner's study (15 s to $30 \mathrm{~min}$ vs. $1-5 \mathrm{~min}$ ), and we do consider many intermediary scales, contrary to Jaffrain's study. Moreover, most $Z-R$ scatterplots fitted here are the whole scatterplots of the series, not those of specific selected storms. Even though different weather regimes may occur during the duration covered by the series, this is not a limitation since (1) this fit will favor the most typical weather regimes in the location of the disdrometer, and (2) the theoretical framework is a statistical one, which is expected to be valid for long time periods. Yet, we also propose a study intended to remove microphysical variability by using relationships involving DSD normalization parameters (see section 6). Another remark is that (like in Steiner and Jaffrain's papers) we only use disdrometer data, which is not strictly equivalent to the use of radar data: here, we compute reflectivity factors and rain intensities from ground-based measurements). Moreover, only time scales are considered in the empirical study. However, the conclusions drawn from the empirical study should apply in space also since the statistical framework considered in the theoretical sections as a 
justification of $Z-R$ scale dependency has been proved to be valid by many empirical studies of radar-rainfall maps (see section 2.3 for more details and references).

[11] The rest of the paper will follow this outline. In section 2, we recall theoretical notions of statistical scaling and of the multifractal theory. In section 3 , consequences of these properties, assumed valid for both $R$ and $Z$, are derived for $Z-R$ relationships. The data set and the empirical methodology are presented in section 4 , and the results are detailed in section 5 . In section 6 , the previous study is refined from the use of normalized $Z-R$ relationships (i.e., involving normalization parameters of the DSD). An overall discussion of the consequences of $Z-R$ parameters scale dependency is proposed in the last section.

\section{Scaling and Multifractals}

\subsection{Scaling}

[12] Statistical scaling is a property shared by many processes and random fields. Such processes are characterized by the existence of such symmetries relating the process aggregated at different scales. Generically, the energy density at two different wave numbers/frequencies will differ from a multiplicative factor that depends only of the scale factor. This property results in power-law energy spectra:

$$
E(k) \approx k^{-\beta}
$$

or

$$
E(\omega) \approx \omega^{-\beta}
$$

where $\omega$ is the time frequency, and $k$ the space wave number.

[13] For instance, standard and fractional Brownian motions are scaling processes. Many financial or geophysical processes also follow such laws, mainly on an empirical basis. However, there are several examples where a theory supports them. In particular, in the field of statistical mechanics of turbulence, Kolmogorov [1941] established a scaling law for velocity increments of a (3D isotropic) turbulent field associated with a $5 / 3$ power-law energy spectrum. A related scaling law (with same spectral exponent) has been demonstrated by Obukhov [1949] and Corrsin [1951] for the concentration of a passive scalar advected by a turbulent field.

\subsection{Multifractal Cascades}

[14] Power/energy spectra are second-order statistics of processes as well as autocorrelations and variograms. Hence, equations (2)-(3) above express a scaling property of a specific (second) order of moment. However, statistics of other orders may also have a kind of scaling behavior. For some of these processes, the knowledge of the single spectral exponent is sufficient to know the scaling exponents of all other statistics from a simple linear relationship: these processes are called "monofractal." This is the case of fractional Brownian motions or other "simple scaling processes" [Lovejoy and Schertzer, 1995].

[15] However, many processes considered in geophysics cannot be considered as monofractal due to their high dynamic range and their "multiplicative" structure [Lovejoy and Schertzer, 2007]. For such processes, called "multifractal", statistics of different orders follow scaling laws whose exponent depends of the order of moments.

[16] Multiplicative cascades, introduced in the turbulence theory [Novikov and Stewart, 1964; Yaglom, 1966] are the conceptual tool for modeling stochastic processes and fields with multifractal statistics. In a few words, multiplicative cascades are an iterative construction based on a series of grid scale refinements associated with i.i.d. random multiplicative modulations. These constructions, which can be either discrete or continuous in scale, can be used to simulate multifractal fields. Depending on the nature of the random modulations and on the discrete or continuous definition of scale, various multifractal models have been defined [e.g., Schertzer and Lovejoy, 1987; She and Levêue, 1994; Dubrulle, 1994].

[17] Multifractal cascades are characterized by scaling laws relating statistical moments and the resolution. In order to follow usual notations in the multifractal literature, the resolution (denoted $\lambda$ ) is conventionally defined as the converse of the scale of interest: in the space domain, $\lambda=L_{\text {out }} l l$ where $l$ is the space scale, and $L_{\text {out }}$ is a constant "outer scale" associated with the largest scale of interest, for instance, planetary scale [e.g., Lovejoy et al., 2008]. A similar definition holds for time resolutions.

[18] A multifractal cascade may be viewed as a random process/field with different descriptions at different resolutions. Hence, the notations involve not only the name of the process (in this section, $\Phi$ ), but also the resolution at which it is defined (or observed), thus the notation $\Phi_{\lambda}$. Moreover physical and mathematical constraints usually require that the mean of the cascading process is conserved across scales: $\left\langle\Phi_{\lambda}\right\rangle=\langle\Phi\rangle$ (in the following, $\langle$.$\rangle denotes statis-$ tical averaging).

[19] Using these notations [e.g., Lovejoy et al., 2008], the fundamental equation of the multifractal formalism is the following:

$$
\left\langle\Phi_{\lambda}^{q}\right\rangle=\lambda^{K_{\Phi}(q)}\langle\Phi\rangle^{q}
$$

[20] This equation expresses that for any positive $q$, the $q$-th order statistical moment of the field follows a power law of the time/space resolution. The exponent of the scaling law, denoted $K_{\Phi}(q)$, depends on the order $q$. By considering all possible values of $q$, we obtain the "moment scaling function" which implicitly contains the statistics if the process or field at all scales of interest. This function has the trivial values $K_{\Phi}(0)=0$ and $K_{\Phi}(1)=0$ (the latter for random fields with a statistical mean which is conserved scale by scale). Moreover, it may be demonstrated that $K_{\Phi}$ $(q)$ is also a convex function [Lovejoy and Schertzer, 2013, appendix 3A].

[21] Realistic variables (in geophysics) can only be represented by continuous in scale multiplicative models for which the choice of the distributions of multiplicative increments is strongly constrained [Schertzer and Lovejoy, 1997]. Among the possible solutions, two of them received a great attention in the geophysical literature: the "Universal Multifractal" model [Schertzer and Lovejoy, 1987], based on log-stable modulations, and the logPoisson cascade model [She and Levêque, 1994; Dubrulle, 1994]. For both models, the convex, nonlinear function $K_{\Phi}$ 
$(q)$ is fully parameterized by the knowledge of two multifractal parameters. They respectively take the forms:

$$
K(q)=\frac{C_{1}}{\alpha-1}\left(q^{\alpha}-q\right)
$$

and

$$
K(q)=\gamma^{+} q+c\left[\left(1-\frac{\gamma^{+}}{c}\right)^{q}-1\right]
$$

[22] For the UM model, $C_{1}$ (comprised in the interval $[0, D]$ for $D$-dimensional processes) may be viewed as an inhomogeneity index for the distribution of the mean level of the field, and $\alpha$ (comprised in [0,2]) is a multifractality index that describes how rapidly inhomogeneity changes with a shift in intensity threshold. For the log-Poisson model, $\gamma+$ is representative of the scaling of the maxima of the process, while $c$ describes the scarcity of such maxima.

[23] Due to Wiener theorem, such cascade models can only produce fields with spectral exponent $1-K(2)<1$. However, other possibly nonstationary models can be derived from the previous by adding a fractional integration to the process (i.e., mainly a power-law $k^{-H}$ filtering). The fractional integration preserves the power-law scaling, which must be, however, interpreted now as a scaling of increments. For instance, the fractional integration of a UM model is referred as the Fractionally Integrated Flux (FIF) model [Schertzer and Lovejoy, 1987].

\subsection{Scaling Properties of Rainfall}

[24] The models presented above have been shown to fit accurately the statistics of many geophysical fields over a wide range of scales [Lovejoy and Schertzer, 2007, 2010]. In particular, the statistics of velocity increments in the atmosphere follow such statistics in a way that confirms and refines Kolmogorov's predictions. The existence of scaling and (or) multifractal properties for rainfall both in space and time has been advocated for a long time [Tessier et al., 1993; Fraedrich and Larnder, 1993; Lovejoy and Schertzer, 1995; de Lima and Grasman, 1999; Pathirana et al., 2003; Lovejoy et al., 2008; de Montera et al., 2009; Verrier et al., 2010; Lovejoy et al., 2012; Gires et al., 2012]. A recent review may be found in Verrier et al. [2011].

[25] The latter study confirmed and extended previous conclusions on the time scaling regimes of rain [Fraedrich and Larnder, 1993; Fabry, 1996]; three main regimes could be distinguished:

[26] 1. For time frequencies smaller than $\sim(3 \text { days })^{-1}$, the spectrum is flat.

[27] 2. For high frequencies $\left(>(30 \mathrm{~min})^{-1}\right)$, the spectrum follows a $\omega^{-1.55}$ power law, and the rainfall process is accurately described by a FIF model.

[28] 3. Intermediate frequencies have an intermediate spectral exponent and could be described by UM cascades.

[29] The detailed interpretation of such regimes will be provided in another study with different data [Rysman et al., 2013].

[30] An interesting feature of the cited studies is that not only rainfall rates (considered in most of these studies) but also radar reflectivities of rainfall events seem to follow multifractal statistics-see the study of TRMM reflectivities by Lovejoy et al. [2008], or the ground-based radar case study by Tessier et al. [1993]. This means that in comparable space and time scaling regimes, properties expressed by equation (4) should hold both for rain rates and radar reflectivities (and then, for reflectivity factors). The next section investigates how such statistics interfere with the existence of $Z-R$ relationships.

\section{From Scaling Properties of Rain to Scale-Dependent $Z-R$ laws}

[31] The theoretical study of the properties of cascades elevated to a (positive) power has been performed by some works, especially devoted to the design of methods of multifractal analysis [Tessier et al., 1993], and some important results have been found about multifractal parameterization of $Z$ and $R$ fields [Tessier et al., 1993; Lovejoy et al., 2008]. In this section, we follow the approach proposed by these authors, in order to investigate the constraints linking moment structure functions and multifractal parameters of both variables. From these constraints (which are discussed in a wider framework than in previous papers, including the up to now neglected log-Poisson case), the scale properties of $Z-R$ parameters are derived.

[32] Let us assume that $Z$ and $R$ follow multifractal statistics over a given space (or time) scale range, with conserved mean. Let us also write a possibly scale-dependent $Z-R$ law:

$$
Z_{\lambda}=a R_{\lambda}^{b}
$$

where $: a=a(\lambda) \quad$ and $b$ assumed constant with scale.

[33] Observable geophysical variables differ from cascade products in the sense that they are spatial averages ("dressed" cascade, Schertzer and Lovejoy [1987]) of a top-down cascade constructed down to smaller scales. Such "bare" and "dressed" cascades generally differ only slightly (except for extreme values). First, take the $b$-th power of $R$ at a given scale and then perform a spatial averaging may introduce small-scale breaks in the scaling. However, the latter are corrected by the averaging steps, justifying the scaling of dressed reflectivities, with $b$ constant across scales (equation (8)) [see Tessier et al., 1993, §3 on similar issues].

[34] Let us take $q$-th and first-order moments on both members of equation (7):

$$
\begin{gathered}
\left\langle Z_{\lambda}^{q}\right\rangle=a^{q}\left\langle R_{\lambda}^{b q}\right\rangle \\
\left\langle Z_{\lambda}\right\rangle=a\left\langle R_{\lambda}^{b}\right\rangle .
\end{gathered}
$$

[35] The parameter $a$ may be eliminated by combination of both equations:

$$
\langle Z\rangle^{q} /\left\langle R_{\lambda}^{b}\right\rangle^{q}=\left\langle Z_{\lambda}^{q}\right\rangle /\left\langle R_{\lambda}^{q b}\right\rangle
$$


[36] Now, apply the fundamental equation of the multifractal formalism (4) for both $R$ and $Z$ (in equation (10)). After simple calculations with evident simplifications of the "means" $\langle Z\rangle$ and $\langle R\rangle$, it appears that the moments scaling functions $K_{Z}(q)$ and $K_{R}(q)$ are related as follows:

$$
K_{Z}(q)=K_{R}(b q)-q K_{R}(b)
$$

[37] This functional relation should hold for positive (real) values of $q$ (except possibly for very high orders for which the moments may be not defined, see [Schertzer et al., 2002] for details).

[38] Tessier et al. [1993] derived an equation identical to equation (11) in the larger framework of powers of any orders of any multiplicative cascades. They also considered the case of $Z-R$ relationships within the framework of Universal Multifractals (see equation (5)) and highlighted that in this case a simplification of equation (11) could be found,

$$
K_{Z}(q)=b^{\alpha} K_{R}(q)
$$

leading to the following relationships between the universal parameters of $Z$ and $R$ [Lovejoy et al., 2008]:

$$
\begin{gathered}
\alpha=\alpha_{R}=\alpha_{Z} \\
C_{1 Z}=b^{\alpha} C_{1 R} .
\end{gathered}
$$

[39] In addition to the results found by Tessier et al., we may emphasize that similar remarks hold for log-Poisson cascades (see equation (6)). In this case, the log-Poisson parameterization changes as follows from $R$ to $Z$ :

$$
\begin{gathered}
c_{Z}=c_{R}=c \\
\gamma_{Z}^{+}=c\left[1-\left(1-\frac{\gamma_{R}^{+}}{c}\right)^{b}\right] .
\end{gathered}
$$

[40] Thus, UM and log-Poisson multifractals seem appropriate for simultaneous characterization of multiple rainfallrelated fields. Indeed, for these parameterizations of $K(q)$, the apparent nondependence on $b$ in 1.h.s. of equation (11) can be taken into account by a well-defined and unambiguous transformation in the parameterization of $K(q)$ (equations (13)-(16)).

[41] Let us now rewrite equation (10) in a slightly different form:

$$
\left\langle Z_{\lambda}^{q}\right\rangle /\langle Z\rangle^{q}=\left\langle R_{\lambda}^{b q}\right\rangle /\left\langle R_{\lambda}^{b}\right\rangle^{q}
$$

valid for all positive $q$, which is equivalent to the following relationships (in distribution) between random variables:

$$
Z_{\lambda} /\langle Z\rangle=R_{\lambda}^{b} /\left\langle R_{\lambda}^{b}\right\rangle
$$

[42] Finally, combining equations (18) and (7) provides the following scaling property:

$$
a(\lambda)=\langle Z\rangle /\langle R\rangle^{b} \lambda^{-K_{R}(b)} .
$$

[43] In terms of spatial and temporal scales, this means that the exponent $a$ should follow a power law with the scale of aggregation $l$ (or $\tau$ ):

$$
\begin{gathered}
a(l) \propto l^{K_{R}(b)} \\
b(l)=b=\mathrm{const} \\
a(\tau) \propto \tau^{K_{R}(b)} \\
b(\tau)=b=\mathrm{const}
\end{gathered}
$$

(as noted above, $b$ is expectedly constant across scales).

[44] In order to ensure self-consistency, it may be seen that this scaling relation form is independent of the choice of the "explicative" variable. This arises form an implicit symmetry in equation (11). Let us substitute in the latter: $q \leftarrow q / b$ :

$$
K_{R}(q)=K_{Z}(q / b)+q / b K_{R}(b)
$$

[45] Since $K_{R}(1)=0$, the special case $q=1$ gives:

$$
b K_{Z}(1 / b)=-K_{R}(b)
$$

which may be reported in equation (21):

$$
K_{R}(q)=K_{Z}(q / b)-q K_{Z}(1 / b)
$$

[46] The equation obtained by dressing the field $Z^{1 / b}$ instead of $R^{b}$ is formally similar to equation (11), leading to the same scaling law as equation (19).

[47] However, there may be in practice a numerical difference between the scaling exponent $K(b)$ involved here compared to that deduced from $Z=a R^{b}$, since the exponent $b$ estimates will be practically sensitive to the methodology of regression. This will be discussed in section 5 .

[48] In equation (20), it is important to see whether it means that $a$ increases or decreases with the scale. This clearly depends on the sign of the $K_{R}(b)$ exponent. Since the $K(q)$ function is convex and has zeros at $q=0$ and $q=1$, $K_{R}(b)<0$ for $b<1$ while $K_{R}(b)>0$ for $b>1$. This means that in the usual case in radar-rainfall estimation, i.e., $b>1$, the coefficient $a$ will be increasing with scale. This is fully coherent with the empirical findings of the papers mentioned in introduction [Morin et al., 2003; Steiner and Smith, 2004; Jaffrain and Berne, 2012).

[49] It should be highlighted that the theoretical approach exposed above could virtually be applied for any other precipitation-related fields as long as they follow multifractal statistics on a given range of scales, which is likely. For instance, the conclusions of this section could be extended to $R-K_{\mathrm{dp}}$ laws used in polarimetric radar-rainfall estimation: $R=c K_{d p}^{d}$ (with $K_{\mathrm{dp}}=$ specific differential phase shift). The lack of empirical proof of multifractality of $K_{\mathrm{dp}}$ prevents to affirm it; however, it seems very likely due to the multiplicative structure of multifractal fields (taking power laws of the latter gives another multifractal field), which is the idea behind the demonstration.

[50] If laws of the form $c \propto l^{K(d)}$ and $d(l)=$ const hold for $R-K_{\mathrm{dp}}$ relationships, then the scaling exponent $K(d)$ involved could be closer to 1 than the $K(b)$ exponent of $Z-R$ relationships used for conventional radars, since $d$ has often been suggested to be closer to 1 than $b$ [e.g., Sachidananda and 
Zrnic, 1987] (see, however, Illingworth and Blackman [2002] for a detailed discussion). This means that due to the fact that polarimetric $R-K_{\mathrm{dp}}$ relationships are generally closer to proportionality than their $Z-R$ alternatives, the polarimetric estimation of rainfall rates should be more robust with respect to scale change. Therefore, the errors in rainfall estimation due to scale effects should be lower in the polarimetric case than in the conventional case. This is totally coherent with the empirical study by Jaffrain and Berne [2012] (Figure 5 of the cited paper), who indeed found a smaller "scale" error in rainfall amounts by using the $R-K_{\mathrm{dp}}$ relationships.

\section{Data Set and Methodology}

[51] In the following sections, the empirical study is restricted to the relationships relating the rain rate $R$ and the reflectivity factor $Z$. The data set considered here consists of the measurements of the DBS instrument [Delahaye et al., 2006] carried out in Palaiseau, France from July 2008 to July 2010. From this instrument, which collects raindrops diameter, fall speed, and time of arrival, high-resolution series of $R$ and $Z$ may be computed from their DSD definitions, with a low noise up to $15 \mathrm{~s}$ integration time. The detailed statistical analysis of the rain rate series (involving tools of the multifractal framework) has been presented in another paper [Verrier et al., 2011]. We refer the reader to this paper for a detailed presentation of the characteristics of the series. It should, however, be precise that the present series differs from the previous ones by a slightly different filtering of nonrain "drops" measurements.

[52] In order to eliminate small rain rates that will not be observed by most instruments (rain gauges and radars), we applied a threshold at $\mathrm{R}=0.1 \mathrm{~mm} / \mathrm{h}$ (at maximal $15 \mathrm{~s}$ resolution). This conventional threshold is also chosen in order to maintain the coherence with a previous study [Verrier et al., 2011] where we worked with a similar threshold and demonstrated the existence of multifractal properties over specific scaling regimes. Note that in the case of disdrometer measurements, there are many measurements of small rainfall rates (and reflectivity factors); the threshold may impact $Z-R$ scatterplots, hence some $Z-R$ regressions methodologies (see below).

[53] Then, the $Z$ and $R$ series have been degraded using integration times that are multiples of the initial resolution of $15 \mathrm{~s}$. The scale factors [i.e., $\lambda=$ scale $/(15 \mathrm{~s})$ ] involved start from 2 to 256 (i.e., 64 min resolution, $\lambda=2^{8}$ ) were chosen so that they are equally spaced in $\log _{2}$ space (i.e., $\operatorname{Int}\left(2^{n / 2}\right)$ with integer $n$ ). Thus, various coarse resolution series of rain rates and reflectivity factors are obtained. From the latter, a scatterplot of strictly positive values of $Z$ and $R$ is found and may be used as a basis for estimating the statistically optimal parameters $a$ and $b$ of equation (1).

[54] At this point, several regression methodologies may be considered, and they are not strictly equivalent. The simplest way to proceed is to perform linear regressions over the scatterplots of the logs of the variables (for $R>0$, $Z>0$ ), in order to linearize equation (1). However, even in this case, the parameters $a$ and $b$ will be sensitive to the choice of the explicative variable, since the regression will not minimize the same criterion. So $\log Z$ vs. $\log R$ will not provide the same estimates $\log R$ vs. $\log Z$, and the departure is generally significant. The latter regression is expected to be more appropriate in the case of radar-rainfall estimation. An intermediate solution would be also to consider the line associated with the principal axis of the log-log scatterplot. In this case, there is no explicative variable and in the case of principal axis of normalized PCA, the slope estimate will be the geometrical mean of that of the previous linear regressions.

[55] Another possibility is to use nonlinear regressions directly on the $Z-R$ scatterplot, without taking the logs. This methodology differs from the previous ones since due to the absence log transformation, these are directly the absolute errors (in the mean square sense) that are minimized, not the relative errors. Therefore, nonlinear regressions will provide a better fit of the high values of $R$ and $Z$, leading to more precise rainfall accumulations [Campos and Zawadzki, 2000]. Levenberg-Marquardt methods are appropriate for such kinds of regression [Levenberg, 1944; Marquardt, 1963].

[56] In the present study, different methods of regression have been applied to the data, summed up by the following nomenclature:

[57] $\mathrm{R}_{1}$ : Linear regression $\log Z=f_{1}(\log R)$

[58] $\mathrm{R}_{1}{ }^{\prime}$ : Linear regression $\log R=g_{1}(\log Z)$

[59] $\mathrm{R}_{2}$ : Principal axis in the $\log R, \log Z$ space

[60] $\mathrm{R}_{2}$ : Principal axis in the centred normalized $\log R, \log$ $Z$ space (both logs shifted to 0 mean and divided by their standard deviation)

[61] $\mathrm{R}_{3}$ : Nonlinear regression $Z=f_{3}(R)$

[62] $\mathrm{R}_{3}{ }^{\prime}$ : Nonlinear regression $R=g_{3}(Z)$

[63] $\mathrm{R}_{4}$ : Bin averages of data and then linear regression log $Z=f_{4}(\log R)$

[64] $\mathrm{R}_{4}{ }^{\prime}$ : Bin averages of data and then linear regression $\log R=g_{4}(\log Z)$

[65] For $\mathrm{R}_{4}$ and $\mathrm{R}_{4}{ }^{\prime}$, the regressions are not performed on the scatterplots of data but of the logs of data averaged over logarithmically equally spaced bins in $R$. This methodology is added in order to fit the conditional expected values of $Z$ over small intervals of $R$ (the bins have a 0.1 width in logarithmic space), in order to highlight "typical" $Z-R$ relationships instead of individual departures to them (due to for instance, to the specific microphysical properties of some storms).

[66] In order to quantify the quality of the regressions, the following statistical indicators have been estimated: the $\mathrm{R}^{2}$ for log-log linear regressions, the percentage of variance (inertia) (\%Iner) explained by the first principal axis for PCAinspired regressions, and also the percentage of "explained" variance for nonlinear regressions (here also denoted by analogy as $\mathrm{R}^{2}$ ). These quality indicators are estimated on the whole scatterplots (but on "binned" data for regressions $\mathrm{R}_{4}\left({ }^{\prime}\right)$ ).

[67] The distinction established between the different regression methodologies helps to focus on the multiscale behavior of $Z-R$ parameters for each of them: if similar laws are found for several methodologies, this supports this idea of a scale dependence of $Z-R$ parameters that is mainly due to the variability of the process (as predicted in section 3 ), not to methodological artefacts. Moreover, observational errors are low for the DBS data, which eliminates another possible artefact source of scale effects (such effects were already considered in cited Morin et al.'s paper).

\section{Synthesis of Empirical Results}

[68] For illustration purposes, the scatterplot of strictly positive values of $Z$ and $R$ of the DBS series at 30 s resolution 


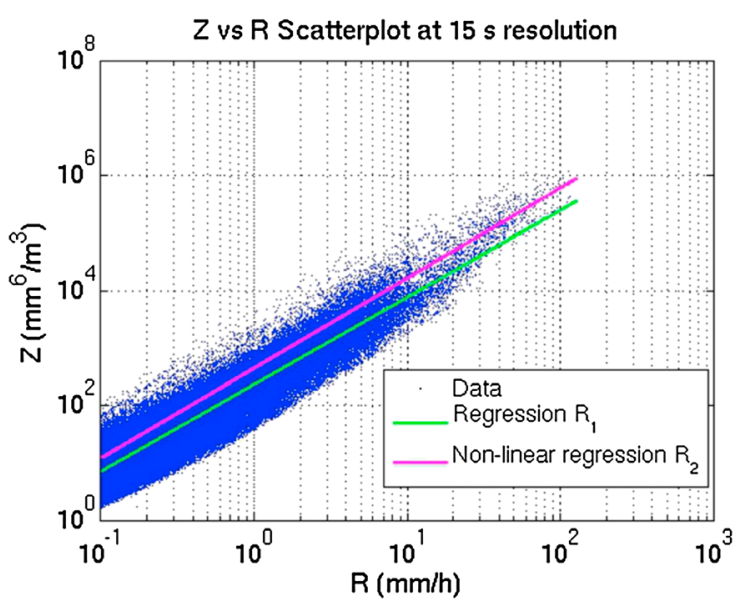

Figure 1. Scatterplot $\log Z$ vs. $\log R$ in logarithmic coordinates and optimal fits for regressions methodologies $R_{1}$ and $\mathrm{R}_{3} . \mathrm{R}_{1}$ corresponds to linear regression $\log Z=f_{1}(\log R)$, while $\mathrm{R}_{3}$ is the nonlinear regression $Z=f_{3}(R)$.

is given on Figure 1. The optimal regression curves for $R_{1}$ and $R_{3}$ regression strategies are shown on the figure, highlighting the non equivalence between both methods. As expected, $\mathrm{R}_{3}$ provides better fits for high values. As explained in the previous section, such fits are performed for multiple integration times and for the methodologies listed above. To give a synthesis of the results, four graphics are provided for each regression method:
[69] 1. The plot of the prefactor $a$ as function of the $\log _{2}$ of the scale factor, i.e., $\log _{2}(\lambda)=\log _{2}$ (time scale/15 s).

[70] 2. The plot of the exponent $b$ as function of the $\log _{2}$ of the scale factor

[71] 3. The plot of an indicator of the quality of the regression $\left(\mathrm{R}^{2}, \%\right.$ explained inertia, see section 4$)$ as function of the $\log _{2}$ of the scale factor. Note that in the case of linear regression, the $\mathrm{R}^{2}$ is insensitive to the choice of the explicative variable in abscissa. The same remark holds for inertia percentages of principal axes. This is why on some $\mathrm{R}^{2} /$ inertia graphs, some curves are undistinguishable.

[72] 4. The plot $\log _{2}$ of the prefactor $a$ as function of the $\log _{2}$ of the scale factor. If the prefactor follows a scaling law such as (equation (20)), then ideally a straight line should appear (with slope $\kappa_{a}=K_{R}(b)$ ). A linear regression fit is provided over all the available range of scales $15 \mathrm{~s}-64$ min, except when some points must be excluded: this provides the scaling exponent $\kappa_{a}$ of the prefactor.

[73] All these for graphs have the same abscissa axis that should be read as follows: $x=0$ means $15 \mathrm{~s}, x=1$ means 30 $\mathrm{s}, x=2$ means $1 \mathrm{~min}, \ldots, x=8$ means $64 \min \left(x=\log _{2}(\lambda)\right)$.

[74] Figure 2 presents the results for the regression methodologies $\mathrm{R}_{1}, \mathrm{R}_{1}{ }^{\prime}, \mathrm{R}_{2}, \mathrm{R}_{2}{ }^{\prime}$ performed on the $\log -\log$ scatterplots. Figure 3 synthesizes the results for the regression methodologies $\mathrm{R}_{3}$ and $\mathrm{R}_{3}$ ' (nonlinear regressions). The methodology based on scatterplots averaged by intervals, $\mathrm{i}$. e., $R_{4}$, and $R_{4}{ }^{\prime}$, leads to the results exposed in Figure 4. Complementary to the graphical representation, Table 1 provides a numerical summary of the regressions,
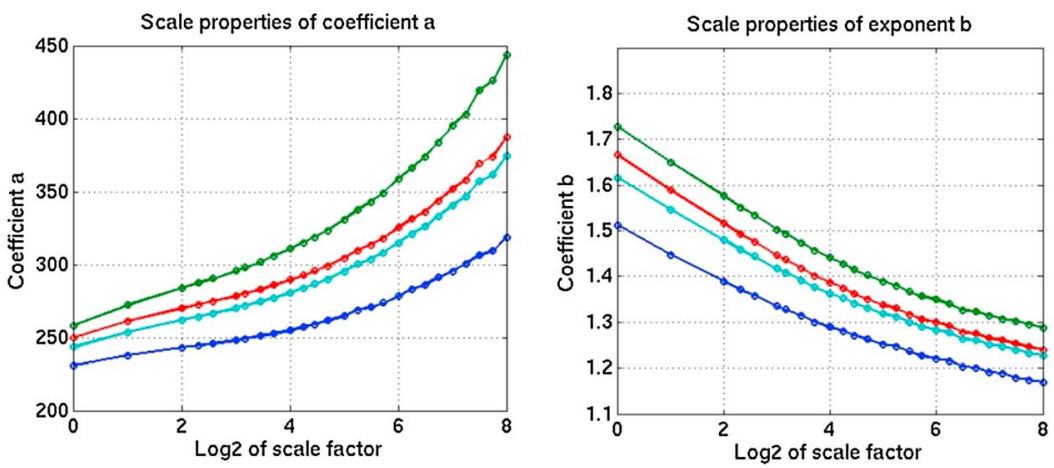

$Z=a R^{b}$ fit:

Linear/pca regressions on log-data
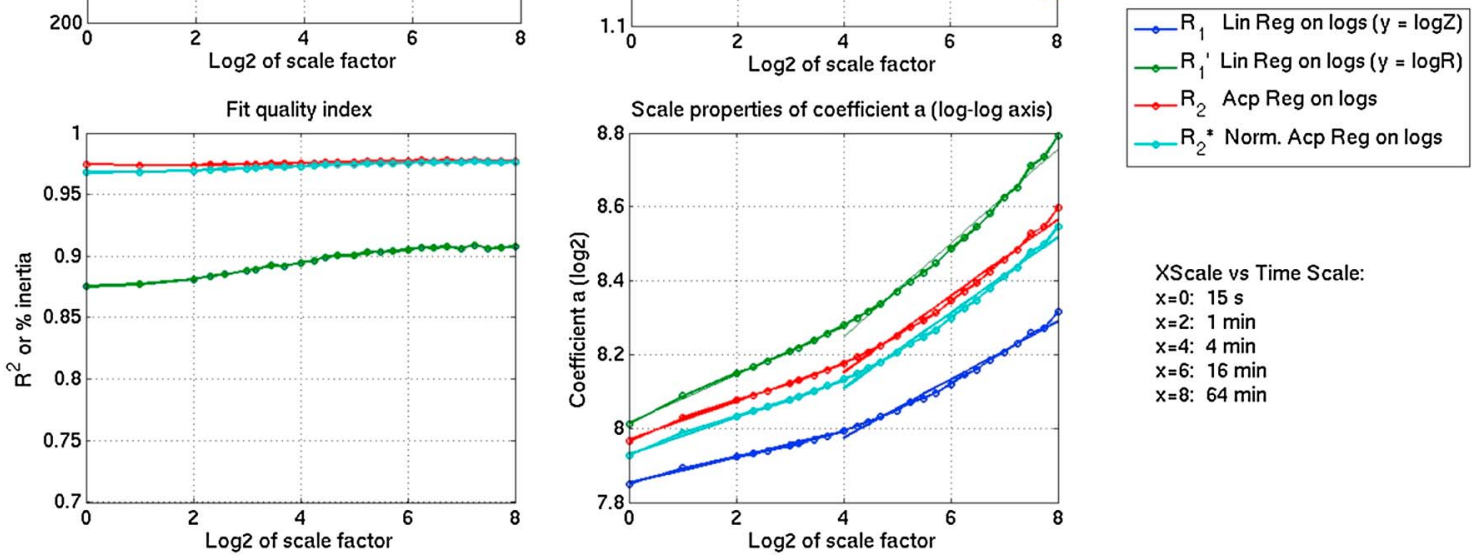

$X$ Scale vs Time Scale:

$\mathrm{x}=0: 15 \mathrm{~s}$

$x=2: 1$ min

$x=4: 4$ min

$x=6: 16 \mathrm{~min}$

$\mathrm{x}=8: 64 \mathrm{~min}$

Figure 2. Estimation of $Z-R$ parameters for linear regression strategies on the $\operatorname{logs}, \mathrm{R}_{1}, \mathrm{R}_{1}{ }^{\prime}, \mathrm{R}_{2}, \mathrm{R}_{2}{ }^{\prime}$ (curves with different colors, see legend). (top left) The estimate of coefficient $a$ as a function of the logarithmic scale factor, i.e., $x=\log _{2}(\mathrm{scale} / 15 \mathrm{~s})$. This means that on the abscissa axis, 0 corresponds to $15 \mathrm{~s}$, etc. (top right) The estimate of exponent $b$ as a function of the same logarithmic scale abscissa. (bottom left) The $\mathrm{R}^{2}$ /explained inertia \% as a function of logarithmic scale factor. (bottom right) The same as Figure 2 (top left) except except that vertical axis is converted in $\log _{2}$ scale. 

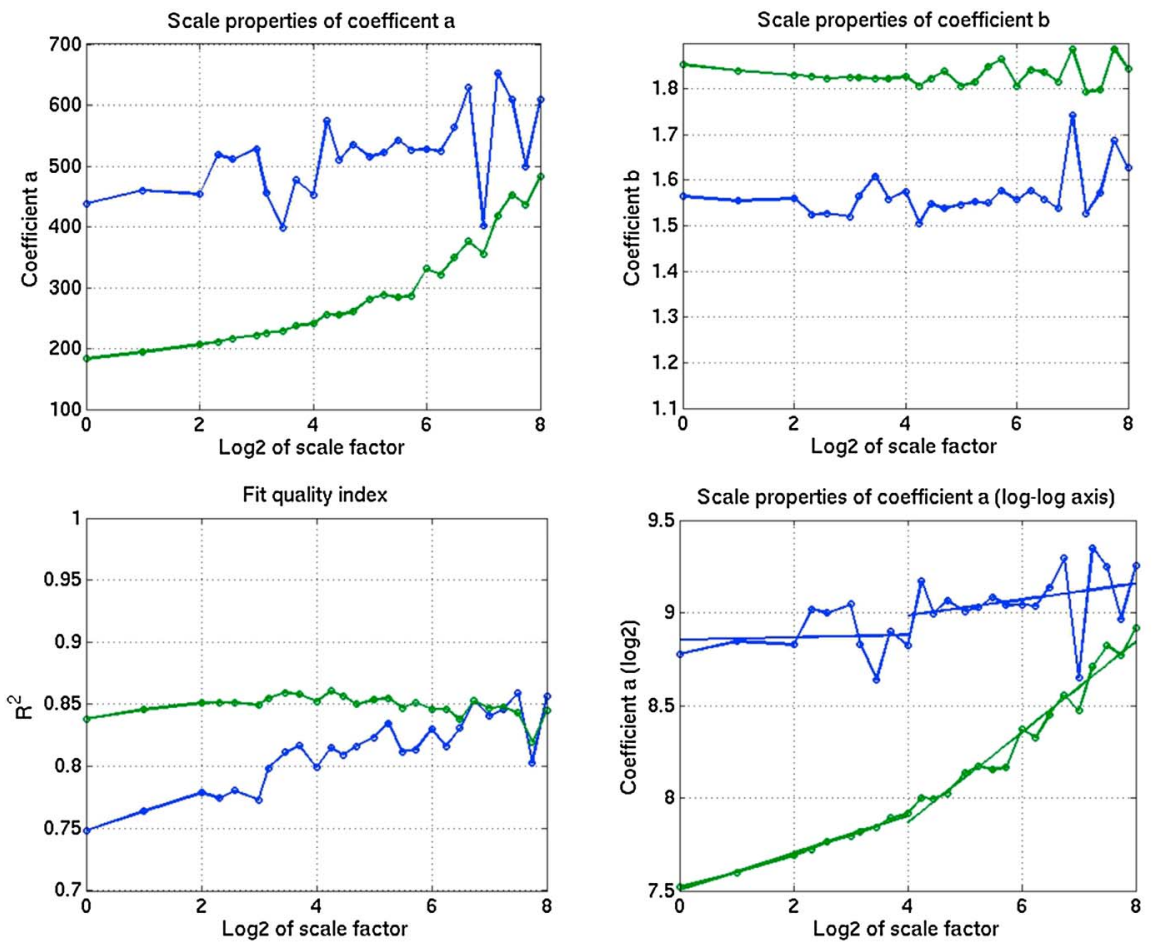

$Z=a R^{b}$ fit

Non-linear fit of data

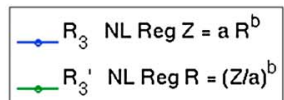

XScale vs Time Scale:

$x=0: 15 \mathrm{~s}$

$x=2: 1 \mathrm{~min}$

$x=4: 4 \mathrm{~min}$

$x=6: 16$ min

Figure 3. Estimation of $Z-R$ parameters for regression strategies $\mathrm{R}_{3}, \mathrm{R}_{3}{ }^{\prime}$ (non linear regressions). Like in Figure 2, the horizontal axis is the $\log _{2}$ of scale factor. The four graphs are organized similarly as in Figure 2.

[75] For all regressions, the prefactor $a$ is (as expected from theory) a growing function of the time scale, generally starting from values $200-250$ at the finest scales up to values in the range $350-600$ at scales close to $1 \mathrm{~h}$. On the contrary, the exponent $b$ can differ significantly from one regression to one another, both in absolute value and scale-dependent behavior. Regressions $\mathrm{R}_{1}\left({ }^{\prime}\right), \mathrm{R}_{2}\left({ }^{\prime}\right)$ lead to scale-dependent $b$ (already observed in Morin et al. [2003]'s study), whereas methodologies $\mathrm{R}_{3}, \mathrm{R}_{3}{ }^{\prime}, \mathrm{R}_{4}$, and $\mathrm{R}_{4}{ }^{\prime}$ support the theoretically expected scale-independent $b$. The latter estimates of the
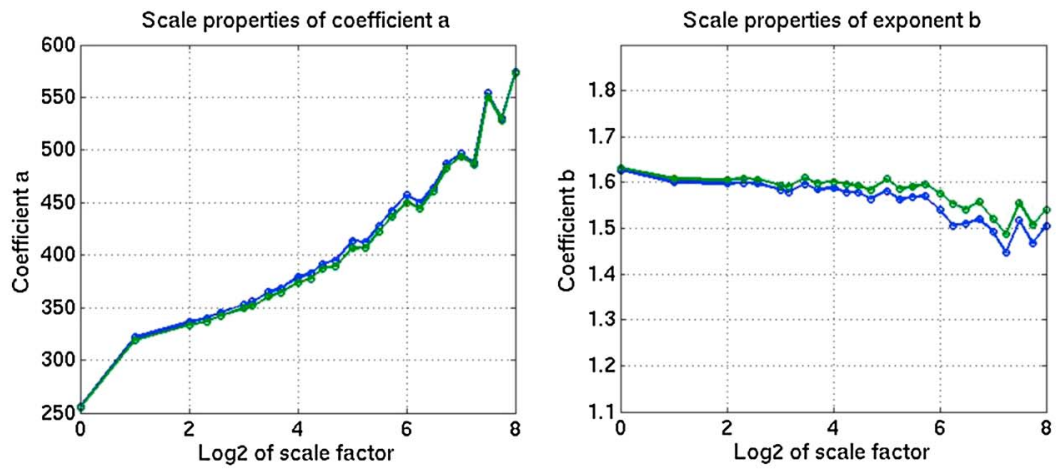

$Z=a R^{b}$ fit:

(Log-)Linear regressions Binned-data
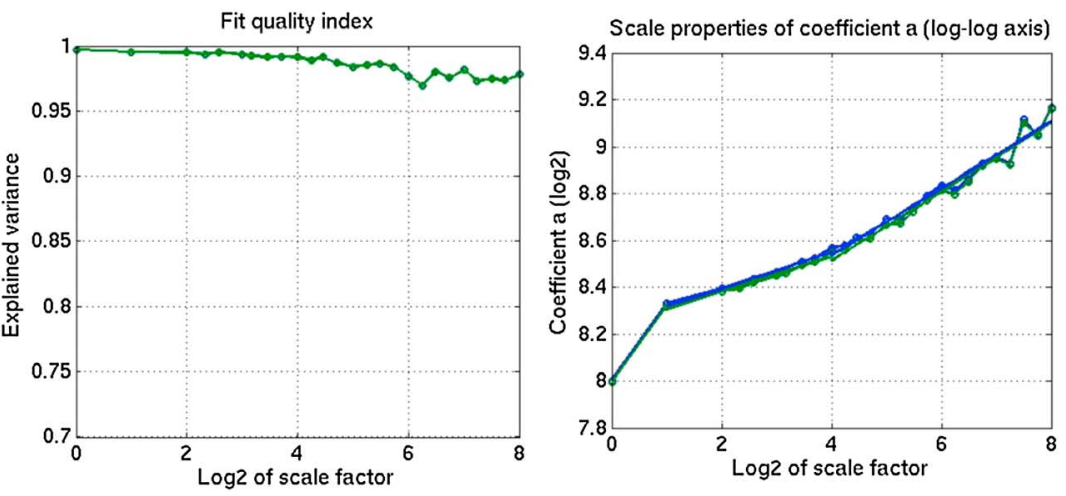

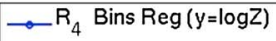

$\rightarrow R_{4}^{\prime}$ Bins Reg $(y=\log R)$

$X$ Scale vs Time Scale:

$\mathrm{x}=0$ : $15 \mathrm{~s}$

$x=2: 1$ min

$x=4: 4$ min

$\mathrm{x}=6: 16 \mathrm{~min}$

$x=8: 64$ min

Figure 4. Same as Figure 3, but for methodologies $\mathrm{R}_{4}, \mathrm{R}_{4}{ }^{\prime}$ (bin averages). 
Table 1. Scaling Coefficients $\kappa_{a}$ of Prefactor $a$ in the Scaling Ranges $15 \mathrm{~s}-4$ Min and 4 Min -64 Min

\begin{tabular}{|c|c|c|c|c|}
\hline Ref. Regression & Description & Ref. Fig. & Range $15 \mathrm{~s}-4 \mathrm{~min}$ & Range $4 \min -64 \min$ \\
\hline R1 & Lin. Reg. $\log Z=f(\log R)$ & Figure 2 & 0.034 & 0.079 \\
\hline $\mathrm{R} 1^{\prime}$ & Lin. Reg. $\log R=f(\log Z)$ & Figure 2 & 0.065 & 0.126 \\
\hline R2 & PCA-based Reg. & Figure 2 & 0.051 & 0.104 \\
\hline $\mathrm{R} 2$, & Norm. PCA Reg. & Figure 2 & 0.049 & 0.102 \\
\hline R3 & Nonlin. Reg. $Z=f(R)$ & Figure 3 & 0.006 & 0.043 \\
\hline R3' & Nonlin. Reg. $\mathrm{R}=\mathrm{f}(\mathrm{Z})$ & Figure 3 & 0.100 & 0.244 \\
\hline R4 & Bin. Reg. $\log Z=f(\log (R))$ & Figure 4 & 0.077 & 0.143 \\
\hline $\mathrm{R} 4^{\prime}$ & Bin. Reg. $\log R=f(\log (Z))$ & Figure 4 & 0.075 & 0.147 \\
\hline R5 & $\log \left(\mathrm{Z} / \mathrm{N}_{0}{ }^{*}\right)=\mathrm{f}\left(\log \left(\mathrm{R} / \mathrm{N}_{0}{ }^{*}\right)\right)$ & Figure 6 & 0.041 & 0.076 \\
\hline $\mathrm{R}^{\prime}$ & $\log \left(\mathrm{R} / \mathrm{N}_{0} *\right)=\mathrm{f}\left(\log \left(\mathrm{Z} / \mathrm{N}_{0} *\right)\right)$ & Figure 6 & 0.046 & 0.083 \\
\hline R6 & $\log Z=f\left(\log \left(\mathrm{R} \mathrm{Dm}^{2.33}\right)\right)$ & Figure 7 & 0.021 & 0.043 \\
\hline $\mathrm{R}^{\prime}$ & $\log \left(\mathrm{R} \mathrm{Dm}^{2.33}\right)=\mathrm{f}(\log (\mathrm{Z}))$ & Figure 7 & 0.023 & 0.045 \\
\hline
\end{tabular}

slopes of $Z-R$ log-scatterplots should, however, be more reliable since these regression techniques are mainly constrained by average and intense values and are practically insensitive to instrumental thresholds contrary to $\mathrm{R}_{1}\left({ }^{\prime}\right), \mathrm{R}_{2}(*)$ regressions which mainly fit on the numerous low and moderate intensities/reflectivities (due to minimization of relative errors).

[76] The most adapted regression technique to the $Z-R$ scatterplot seems to be that based on bin (i.e., conditional) averages, $\mathrm{R}_{4}\left({ }^{\prime}\right)$. It shows the existence of a "typical" linear relationship linking $\log (Z)$ and $\log (\mathrm{R})$ with an excellent $\mathrm{R}^{2}$, associated with the following main features: (1) The exponent $b$ is almost constant (but slightly decreasing) in the range 1.51.6 across scales, and (2) The prefactor $a$ grows as power law of time scale $\tau\left(a(\tau) \sim \tau^{\mathrm{\kappa a}}\right)$ in the two separate scaling regimes $30 \mathrm{~s}-4 \mathrm{~min}$ and from $4 \mathrm{~min}$ to $1 \mathrm{~h}$. As shown in Table 1 , the scaling coefficients $\kappa_{a}=K_{R}(b)$ of the power law are $\sim 0.07$ below $4 \mathrm{~min}$ and $\sim 0.14$ for larger scales.

[77] These conclusions are mainly confirmed by other regressions (excepting the $b$ estimates for $\mathrm{R}_{1}\left({ }^{\prime}\right), \mathrm{R}_{2}\left({ }^{\prime}\right)$ methodologies), which also provide scale-growing values of $a$ that may be modeled by power laws of scale $a(\tau) \sim \tau^{\kappa a}$ in the same scaling regimes identified as above, with a transition at $4 \mathrm{~min}$ scale. For all methodologies, it is found the scale dependency becomes quicker in the second scaling regime with larger $\kappa_{a}$ estimates.

[78] The latter exponent should be compared with the theoretical exponent $\kappa_{a}=K_{R}(b)$ predicted in section 3 . This is not trivial since for high frequencies greater than $(30 \mathrm{~min}-1$ h) $)^{-1}$, the power spectrum of the rain rate series has a spectral exponent $\beta>1$ that prevents the series to be modeled by strictly conservative and stationary multifractal cascades (which was an hypothesis for establishing equation (18)). In fact, the rain intensity series should be viewed as the $H$ fractional integral of a conservative Universal Multifractal flux [Verrier et al., 2011]. In the latter cited paper, it has been found that this flux could be modeled in the range $15 \mathrm{~s}-32 \mathrm{~min}$ by the UM model with optimal parameters $\alpha=1.8$ and $\mathrm{C}_{1}=0.13$ (see equation (5)), but that a 0.4 fractional integral should be added for smallest scales. However, the latter study also showed that the first-order structure function of the rainfall process flattens quickly for lags greater than $\sim 8 \mathrm{~min}$ (i.e., $H$ exponent rapidly decreases to 0 for larger lags). So, even though the hypotheses of the theoretical part of the present paper (section 3) are not strictly speaking applicable in the case of the DBS series, it seems possible to compare the empirical estimate of $\kappa_{a}$ in the second scaling regime $4 \mathrm{~min}-1 \mathrm{~h}$ with the value of $K_{R}(b)$ computed with the UM parameters recalled above. Reporting these values $\left(\alpha=1.8\right.$ and $\left.C_{1}=0.13\right)$ for parameterization of the function $K_{R}(q)$, the values of the theoretical exponent for the scaling of prefactors $K_{R}(b)$ may then be estimated. Using typical values $b=1.5-1.6$ (associated with regressions $\mathrm{R}_{3}\left({ }^{\prime}\right)$ and $\mathrm{R}_{4}\left({ }^{\prime}\right)$ and coherent with many literature estimates), it is found $K_{R}(b)=0.09-0.12$ which agrees well with the values reported in Table 1 . Regarding the regime $15 \mathrm{~s}-4 \mathrm{~min}$, we cannot conclude probably due to the discrepancies associated with the additional $H$ parameter. Physically speaking, the transition at $4 \mathrm{~min}$ identifiable from structure functions and from previous graphs could be linked with the effect of rain/no-rain intermittency, i.e., breaks in the scaling associated with the fractality of rainfall occurrence processes [Verrier et al., 2011].

[79] The overall results of the regressions, and especially those of methodologies $\mathrm{R}_{3}\left({ }^{\prime}\right)$ and $\mathrm{R}_{4}\left({ }^{\prime}\right)$, empirically confirm the notion of scale dependence of $Z-R$ parameters elaborated in section 3 . They are also consistent with the findings of Morin et al. [2003], Steiner et al. [2004], and Jaffrain and Berne [2012] (who, however, mainly considered spatial scales). If the exponent $b$ is constant across scales whereas $a$ increases with integration times, this means that the inadequate use of high-resolution $(a, b)$ parameters at larger scales and on larger integration times will result in an overestimation of rain rates from reflectivity factors using a relation of the form $R=(Z / a)^{1 / b}$. If such a behavior holds in the space domain, this means that similar errors will arise in the estimation of rainfall rates from radar reflectivities. Since radar pixels are large $\left(\sim 1 \mathrm{~km}^{2}\right)$, whereas the parameters $a$ and $b$ are often derived from disdrometer measurements at small scales, there would be an error which would be greater for lower resolutions radar. These ideas will be developed with more details in section 7 .

\section{Normalized $Z-R$ Relationships With the (N0*, Dm) DSD Normalization}

[80] In section 5, the regression methodologies have been applied to the data in order to fit equation (1). Due to the numerous data points in the series which cover a two-year period, the $Z-R$ relationships are only the "typical" climatological ones. As seen in section 5, a great scatter remains in the $\log Z-\log R$ data space. The natural microphysical variability of $Z-R$ relationships is expected to be responsible of a significant part of the scatter and of the low correlation coefficients. But it could not only impact the scatter from the typical 
regression, but the "typical" regression itself. The reason for this is simply that the extreme and nonlinear variability of rainfall processes will be sensitive to aggregation of scales. This was already the same qualitative idea that was underlying the equations exposed in section 3. Hence, in order to estimate more precise $Z-R$ relationships on less scattered data, variants of equation (1) involving normalization parameters of DSD should be considered.

[81] Many normalization techniques have been proposed in the literature [e.g., Sekhon and Srivastava, 1970; Sempere Torres et al., 1994, 1998; Testud et al., 2001; Lee et al., 2004; Lovejoy and Schertzer, 2008]. While former normalization procedures involved one reference DSD moment, Testud et al. [2001] and Lee et al. [2004] have shown improvements led by the use of a second reference DSD moment. However, most existing DSD normalization procedures often rely on unrealistic hypotheses (e.g., homogeneity, absence of scale dependency). On the contrary, the turbulent (multifractal) character of rainfall-related variables states that in fact DSD should be strongly scale dependent. Then, as pointed by Lovejoy and Schertzer [2008], DSD variability should be constrained by controlling macroscopic (scaling) rainfall variables. This means that scaling, macroscopic variables should be involved in normalization procedures. Especially, Lovejoy and Schertzer [2008] reformulated the problem in terms of nondimensionalization. Based on a drop mass distribution formalism, they suggested that turbulent reference variables such as the number density of drops and the liquid water content would provide a more correct nondimensionalization. Indeed, correct DSD normalizations should involve a quantity with a dimension on mass, which is missing in other existing approaches based on questionable homogeneity assumptions (due to implicit conversion between drop volumes and masses using the density of water). In the same paper, the authors found empirical support of their nondimensionalization method based on the study of high-resolution, 3D drop data associated with several storms.

[82] Whatever the limitations associated with some DSD formalisms, we would like to evaluate the behavior of normalized variants of $Z-R$ laws across scales. In the following, attention is focused on the approach proposed by Testud et al. [2001], which is used in practical radar precipitation estimation algorithms such as ZPHI [Testud et al., 2000]. The idea of Testud et al. [2001] is to include in Z-R relationships two additional parameters constructed from specific combination of some moments of the DSD (of course, moments of different orders than 6 and 3.67, associated with $Z$ and $R$, respectively). Within this approach, the DSD is written as:

$$
N(D)=N_{0}^{*} f\left(D / D_{m}\right)
$$

where $\mathrm{D}$ is the raindrop equivalent diameter and $f \mathrm{a}$ "shape" function. $N_{0}^{*}\left(\mathrm{~m}^{-3} \cdot \mathrm{mm}^{-1}\right)$ and $D_{m}(\mathrm{~mm})$ are from the 3-rd and 4-th moments of the DSD.

[83] Let us denote

$$
M_{k}=\int N(D) D^{k} d D
$$

the $k$-th moment of the DSD.

[84] The multiplicative coefficient $N_{0}^{*}$ and the scale parameters are defined as follows:

$$
\begin{gathered}
N_{0}^{*}=\frac{4^{4}}{6} \frac{M_{3}^{5}}{M_{4}^{4}} \\
D_{m}=\frac{M_{4}}{M_{3}}
\end{gathered}
$$

[85] A key point with this normalization is that for any couple of orders $(j, k)$, the adequately normalized moments of the DSD are power law related:

$$
\frac{M_{k}}{N_{0}^{*}}=\left(\frac{M_{j}}{N_{0}^{*}}\right)^{\frac{k+1}{j+1}} \zeta_{k} \zeta j^{-\frac{k+1}{j+1}}
$$

with $\zeta_{k}=\int f(x)^{k} d x$

[86] This behavior is remarkable [Testud et al., 2001] since contrary to $Z-R$ relationships based on Gamma distribution hypothesis (1), equation (28) does not rely on any assumption on the DSD shape and (2) the exponent $b$ of the power law does only depend of the orders $k$ and $j$ involved and not on the shape nor parameters of the DSD.

[87] For $j=3.67$ and $k=6$, the rain rate and the reflectivity factor are related in the following way:

$$
\frac{Z}{N_{0}^{*}}=a^{\prime}\left(\frac{R}{N_{0}^{*}}\right)^{1.5}
$$

[88] Testud et al. [2001] showed the existence of a dual relation involving the normalization parameter $D_{m}$ :

$$
Z=a^{\prime \prime} D_{m}^{2.33} R
$$

[89] The measurements performed by the DBS contain all necessary information on the DSD to estimate the moments $M_{3}$ and $M_{4}$ at $15 \mathrm{~s}$ resolution. The obtained series are then aggregated at coarser time resolutions. Therefore, (equations (26)-(27)) may be applied at multiple resolutions so $N_{0}^{*}$ and $D_{m}$ are available at all scales in the range previously considered. The normalization parameters are reestimated at different resolutions in order to mitigate the problems evoked above about DSD scale dependencies. Of course, the methodology exposed in sections 4 can then be adapted in order to fit the normalized $Z-R$ relationships (equations (29)-(30)).

[90] Additional methodologies are thus defined:

[91] R5: Linear regression $\log \mathrm{Z} / N_{0}^{*}=f_{5}\left(\log R / N_{0}^{*}\right)$

[92] R5': Linear regression $\log R / N_{0}^{*}=g_{5}\left(\log \mathrm{Z} / N_{0}^{*}\right)$

[93] R6: Linear regression $\log Z=f_{6}\left(\log D_{m}^{2.33} R\right)$

[94] R6': Linear regression $\log D_{m}^{2.33} \mathrm{R}=g_{6}(\log Z)$

[95] In the laws to be fitted, with notations

$$
\frac{Z}{N_{0}^{*}}=a^{\prime}\left(\frac{R}{N_{0}^{*}}\right)^{b^{\prime}}
$$

and

$$
Z=a^{\prime \prime}\left(D_{m}^{2.33} R\right)^{b^{\prime \prime}}
$$

it is not assumed a priori that $b^{\prime}=1.5$ and $b^{\prime \prime}=1$, respectively, even though these are the expected values. Of course, the parameter $\mathrm{a}^{\prime}$ is here not the same as the prefactor defined in equation (1); however, it is interesting to investigate whether an empirical scale dependence still holds for it. 

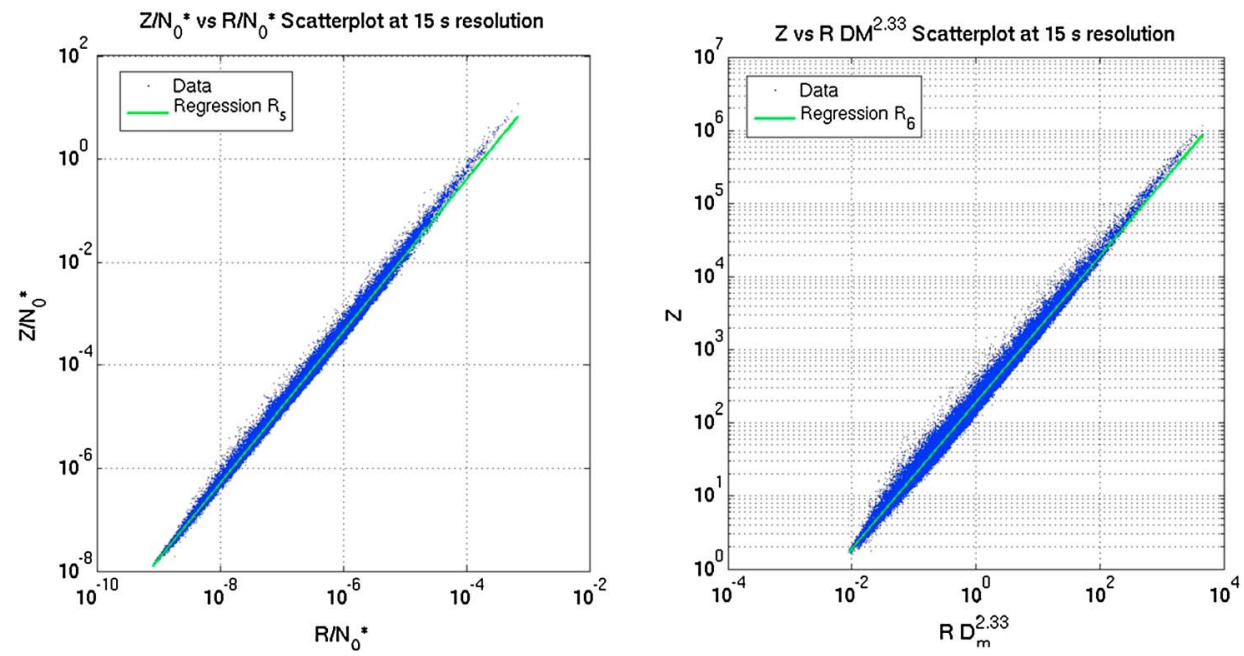

Figure 5. (left) Scatterplot $\log Z / N_{0} *$ vs. $\log R / N_{0} *$ in logarithmic coordinates and optimal fit for regressions methodology $\mathrm{R}_{5}$. R5 corresponds to the linear regression $\log Z / N_{0} *=f_{5}\left(\log R / N_{0} *\right)$. (right) Scatterplot of $\log Z$ vs. $\log \left(R D_{m}{ }^{2.33}\right)$ and optimal fit for regressions methodology $\mathrm{R}_{6}$. R6 corresponds to the linear regression $\log Z=f_{6}\left(\log D_{m}^{2.33} R\right)$.

[96] As shown on Figure 5, the scatterplot of normalized variables is spectacularly less scattered than in Figure 1, with an excellent correlation coefficient. This confirms the interest of the approach proposed by Testud et al. [2001] and subsequent studies: if reliable estimates of $N_{0}^{*}$ and $D_{m}$ are available, the great microphysical scatter in $Z-R$ space is washed out at a fixed scale, leading to robust rainfall rate estimation equations.

[97] However, how do these equations behave across scales? The application of methodologies $R_{5}$ and $R_{6}$, illustrated on Figures 6,7 , is synthetized in Table 1 . The $\mathrm{R}^{2}$ estimates are excellent at all scales $(>0.99)$, confirming the improvement given by the normalization approach. For all scales, the exponents $b^{\prime}$ and $b^{\prime \prime}$ estimates are almost constant with scale and very close to the theoretical values 1.5 and 1 , respectively. On the contrary, the prefactors $a^{\prime}$ and $a^{\prime \prime}$ seem to vary with scale in an increasing way. Coherently with the findings of section 5 , two scaling regimes may be identified separated by a slight transition at 4 min scale. For each of these two regimes, both $a^{\prime}$ and $a^{\prime \prime}$ grow as a power law of scale, and the growth is faster at scales larger than $4 \mathrm{~min}$. Yet, a look at Table 1 shows a significant difference from the study exposed in section 5 . For both scaling regimes, the scaling exponents $\kappa_{a}$ of $a^{\prime}$ and $a$ " are two or three times lower than those found by the fitting of nonnormalized scatterplots. In section 5, we have reported $\kappa_{a}=0.07$ and 0.14 for both scaling regimes while considering the scaling of nonnormalized $Z-R$ relationship prefactor $a$. In the case $N_{0}^{*}$ of normalization, the scaling exponents are reduced to 0.04 and 0.07 , while the $D_{m}$-normalized fits lead to very low scaling exponents $0.02-0.04$. These low exponents are the indicator that normalized $Z-R$ relationships have parameters which are more stable with scale, showing that these $Z-R$ relationships that are more robust to scale artefacts such as those predicted in section 3. This is consistent with some recently published empirical results [Berne et al., 2012].

[98] How to explain this robustness? The latter is a priori nontrivial to demonstrate, since due to the statistical dependence between $R, Z, N_{0}^{*}, D_{m}$, a theoretical multiscale law does not seem to be derived from the multifractal theory. Still, simple considerations may help to understand why the fits of normalized laws (equations (29)-(30)) are more stable across scales. Qualitatively, the increased scale-by-scale robustness of these relationships (compared to those considered in section 5) results from the huge additional information provided by the knowledge of DSD parameters at all scales. To understand this more in detail, let us recall that the DSD has been written as the combination of the two parameters $N_{0}^{*}$ and $D_{m}$ (multiplicative factor and scale parameter) and of a "normalized DSD" function denoted $f$ (see equation (27)). From equation (28), it is evident that the prefactor $a^{\prime}$ in (equation (29)) depends mainly on $\zeta_{6} \zeta_{3.67}^{-1.5}$ with $\zeta_{k}=\int f(x)^{k} d x$. Similarly, it may be shown easily that the prefactor $a^{\prime \prime}$ in (equation (30)) depends mainly on $\zeta_{6} / \zeta_{3.67}$. Hence, it is shown that even though a moderate scale dependence of normalized shape functions seems to exist, the normalization parameters $N_{0}^{*}$ and $D_{m}$ contain an information that is strongly correlated with the scale dependence of nonnormalized $Z-R$ relationships and likely with the underlying extreme, nonlinear, multifractal variability of rainfall.

[99] It has been shown that the addition of the information provided by the parameters $N_{0}^{*}$ and $D_{m}$ may partially resolve the scale dependency of $Z-R$ laws prefactors theoretically derived in section 3 and empirically proven in section 5 . Of course, the use of adequately normalized $Z-R$ laws is strongly constrained in the case of applications (possibly without any disdrometer) by the limited knowledge of $N_{0}^{*}$ and $D_{m}$ over large intervals and over spatial domains. Yet, it should be noted that algorithms like ZPHI [Testud et al., 2000] provide maps of estimates of $N_{0}^{*}$ that could be used in a true radar case study to investigate the scale robustness of such precipitations products.

\section{Impacts and Correction of Scale Artefacts}

[100] The scale dependency of the factor $a$ in $Z-R$ power laws has been empirically verified above by the use of disdrometer data aggregated at different integration times. Yet, such a law is likely to hold also when different spatial resolutions are considered. Theoretically, the scaling law derived in section 3 is valid whenever the hypothesis of multifractality is valid, the latter seeming empirically true for both temporal 

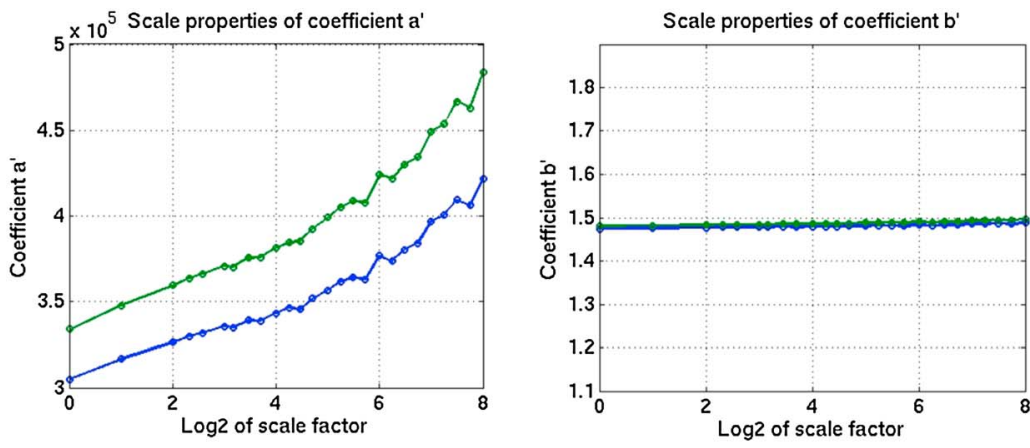

$$
\begin{aligned}
& Z / N_{0}{ }^{*}=a^{\prime}\left(R / N_{0}^{*}\right)^{b^{\prime}} \text { fit: } \\
& (\text { Log)-linear regressions }
\end{aligned}
$$

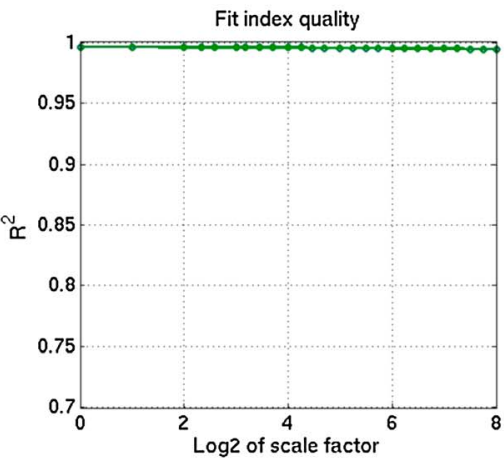

Scale properties of coefficent a' (log-log axis)

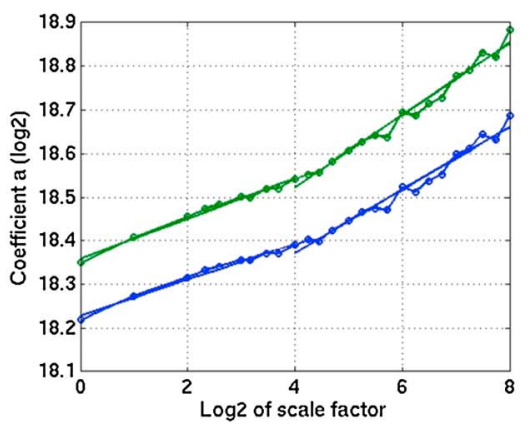

$\rightarrow R_{5}$ Lin Reg on $\operatorname{logs}\left(y=\log \left(Z / N_{0}\right)\right)$

$\ldots R_{5}^{\prime}$ Lin Reg on $\log \left(y=\log \left(R / N_{0}\right)\right)$

XScale vs Time Scale:

$\mathrm{x}=0: 15 \mathrm{~s}$

$\begin{array}{ll}x=2: & 1 \text { min } \\ x=4: & 4 \mathrm{~min}\end{array}$

$x=6: 16 \mathrm{~min}$

$x=8: 64 \mathrm{~min}$

Figure 6. Estimation of $Z / N_{0}{ }^{*}$ vs. $R / N_{0}{ }^{*}$ law parameters for regression strategies $\mathrm{R}_{5}, \mathrm{R}_{5}{ }^{\prime}$. Abscissa is the same as in Figures 2-4. The graphs are organized as in previous figures except that $b^{\prime}$ replaces $b$ and $a^{\prime}$ replaces $a$ (see equation (31)).

and spatial variability. Hence spatial aggregation of scales or changes in pixel size should impact $Z-R$ relationships. This means that radar-rainfall estimation applications will be impacted by biases whenever scale issues are involved. In particular, two typical cases are concerned:
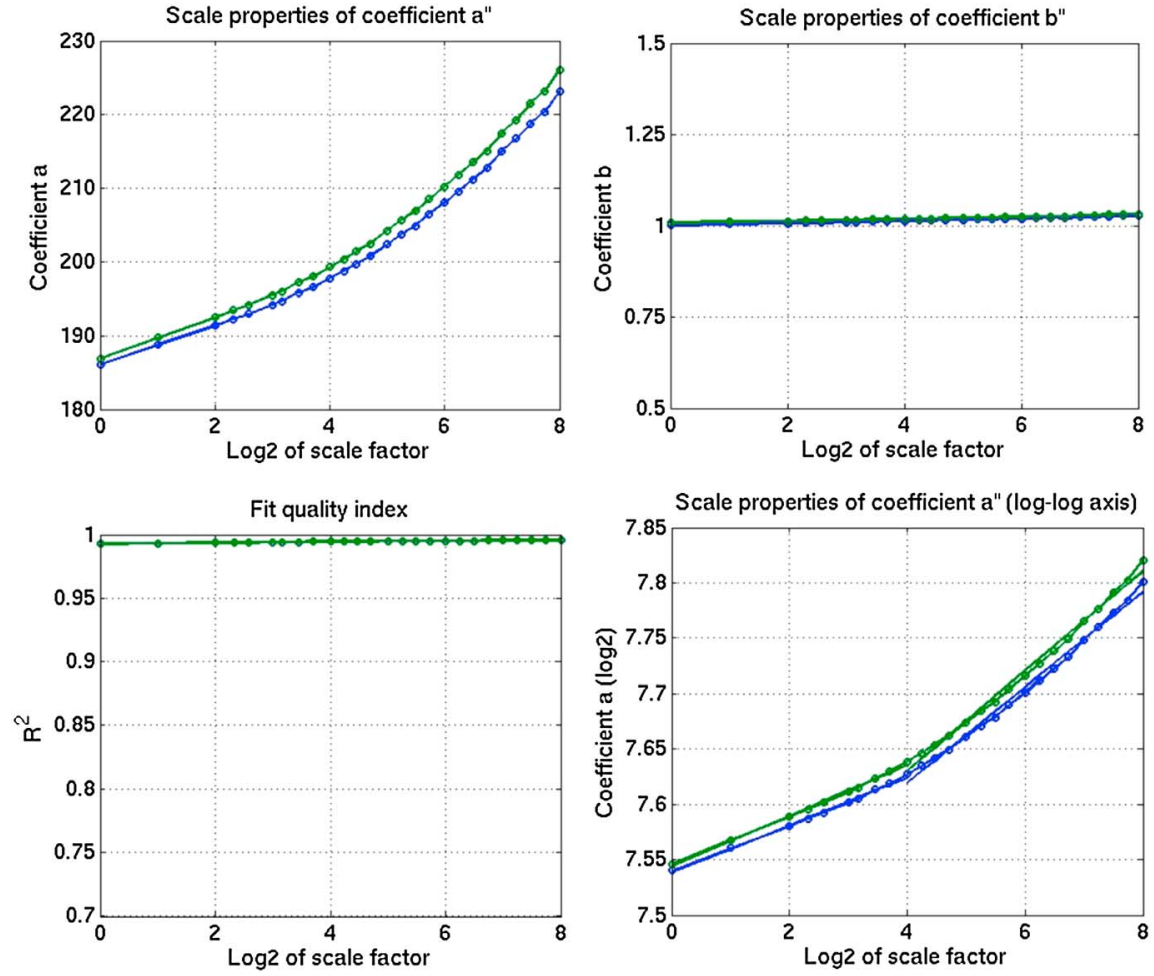

[101] 1. Two distinct radars with different resolutions should use different $Z-R$ relationships. This is especially true when both resolutions differ significantly from each other: for instance the estimation of rainfall intensities from reflectivity data obtained by a ground based radar with a pixel size of

Figure 7. Same as Figure 6 but for regression strategies $\mathrm{R}_{6}, \mathrm{R}_{6}{ }^{\prime}$ and parameters $a^{\prime \prime}$ and $b^{\prime \prime}$ of $Z$ vs. $R D_{m}{ }^{2.33}$ law. 
$500 \mathrm{~m}-1 \mathrm{~km}$ should not use the same $Z-R$ relationships as a spaceborne precipitation radar (e.g., TRMM PR) with a resolution of the order of $4-5 \mathrm{~km}$. This is important to quantify the errors in rain rate estimation while comparing spaceborne radars and ground-based ones since the inversion of spaceborne measurements using higher-resolution ground-based measurements remains a problematic issue.

[102] In the range $1-20 \mathrm{~km}$, rainfall maps seem to follow multifractal properties, even in the interior of rainfall structures; they are characterized by (conservative, UM) multifractal exponents that are the same as those describing time variability in the physically comparable range $1 \mathrm{~min}-30 \mathrm{~min}$ [Verrier et al., 2010, 2011]. This means that the exponent $K(b)$ in equation (19) could be identical in these time and space scale ranges and that a relation of the form $a(l) \propto l^{K(b)}$ may be conjectured at these scales in the space domain. If the parameter $a$ that is valid at $l_{1}$ is applied at a larger scale $l_{2}$, there will be (statistically) an underestimation of $a$ and therefore (for constant $b$ ) an overestimation of the rainfall rate [Steiner and Smith, 2004].

$$
\frac{\hat{R}_{l 2}}{R_{l 2}}=Z_{l 2}{ }^{1 / b(l 2)-1 / b(l 1)} \frac{a\left(l_{1}\right)^{1 / b(l 1)}}{a\left(l_{2}\right)^{1 / b(l 2)}}
$$

In the case of constant $b$, the overestimation will therefore follow a scaling law:

$$
\frac{\hat{R}_{l 2}}{R_{l 2}}=\left(\frac{l_{2}}{l_{1}}\right)^{\frac{K(b)}{b}}
$$

With resolutions considered above, e.g., $l_{1}=1 \mathrm{~km}, l_{2}=5 \mathrm{~km}$, and the typical values $b=1.5$ and $K(b)=0.14$ (see section 5), it is obtained $\frac{\hat{R}_{l 2}}{R_{l 2}} \approx 1.162$, hence $+16.2 \%$ of relative error. The relative error could even be greater in the case of intense storms. Within the latter, the statistical scaling properties are expected to be modified due to increased variability and inhomogeneity. We tried to apply the methodology of section 5 on few selected $1 \mathrm{~h}$ rain events contained in the DBS series (result not shown here) and found slightly greater scaling exponents $K(b)$ that could lead to more than $+20 \%$ of relative error for $a_{1}$ scale factor $\lambda=5$. Note that since $\frac{R_{l 2}}{R_{l 2}}=Z_{l 2}$ $1 / b(l 2)-1 / b(l 1) \frac{a\left(l_{1}\right)^{1 / b(1)}}{a\left(l^{1 / b(l 2)}\right.}$, the relative error slightly depends on rainfall intensity since empirically the exponent $b$ is not in fact a true constant. In section 5 , it has been found that $b$ slightly decreases with scale which means that $1 / b\left(l_{2}\right)-1 / b$ $\left(l_{2}\right)>0$ and therefore the relative error is expectedly more important in the case of intense reflectivities. Hence, intense rainfall rates are more concerned with scale-induced biases in $Z-R$ relationships, with a possible impact on long-term rainfall accumulations estimated from radars.

[103] 2. Since radar sampling volumes widen while increasing the distance from a given radar, measurements are initially distributed over polar grids with pixel area proportional to the distance. Then, it may be understood that "raw" radar measurements in polar coordinates have an equivalent spatial resolution that increases with distance. Since we have shown above that $Z-R$ relationships are not scale independent, this means that the use of a single $Z-R$ relationship in signal processing algorithms of a given radar device will necessary induce significant biases in rainfall estimates even if the radar-rainfall relationship would be "perfectly" calibrated at a specific distance or at some given locations. Opposite, a systematic bias will occur at other distances and especially for the pixels located far away from the radar.

[104] Similar calculations like those of previous paragraph can show that by using a $Z-R$ relationship that is correct at distance $r_{0}$ at another distance $r$, the (statistical) error will take the form of a spurious factor of the form:

$$
\frac{\hat{R}}{R}=\left(\frac{r}{r_{0}}\right)^{\frac{K(b) d}{b}}
$$

where $K(b) \sim 0.1-0.2, b \sim 1.5$, and $d$ is a geometric dimension describing how fast the equivalent resolution increases with the distance (hence, in 3D, $d=2 / 3$ since the radar sampling volume is quadratically dependent on distance). This means that rain rates tend to be overestimated away from the radar (and possibly underestimated very close to it).

[105] 3. An important issue is to improve the retrievement of radar $Z-R$ relationships using collocated raindrop size distribution information. Indeed, DSD data provide information a priori useful for the estimation of optimal parameters in equation (1). However, the spatial resolution of a radar is clearly huge compared to the catchment surface of a disdrometer, the latter leading to "point-scale" DSD estimations and therefore to point-scale estimates of the prefactor $a$ and of the exponent $b$. If $Z-R$ relationships are dependent of the space scale in the same way as above, this would lead to a significant error in the estimation of $a$ and therefore in retrieved rainfall rates. However, relations such as (equation (20)) are not easy to apply directly to model such errors, since (1) there also a difference between the time resolutions of both instruments (radar data consist in an average of a few "instantaneous" scans), and (2) few information on possible multifractality of rainfall at horizontal space scales $<100 \mathrm{~m}$ is available in the literature (due to limited resolution of weather radars); hence, the existence of a law of the form (equation (20)) remains theoretically uncertain (see, however, Lovejoy and Schertzer [2008] for an empirical study).

[106] How to eliminate such practically undesirable biases? For $Z-R$ relationships, this remains a difficult task since this scale dependence seems subtlety constrained by the heterogeneities in microphysical properties. Moreover, the errors described above are statistical, and the spurious factors described above should be understood in this perspective. However, in order to improve rainfall accumulations (which are expected to be representative of statistical averages), we suggest three possibilities that may be used to wash out or diminish scale artefacts in radar-rainfall estimation.

[107] I. A conceptually simple possibility would be to correct expected artefacts of the form $\left(l_{2} / l_{1}\right)^{K(b) / b}$ by simply multiplying estimated rainfall rates by the converse term. With the values estimated in the present studies, this means that if $Z-R$ relations are well calibrated at spatial resolution $l$, then at resolution $l . \lambda$, the outputs in terms of rainfall intensities should by multiplied by $\lambda^{-0.1}$ or $\lambda^{-0.15}$.

[108] II. A second possibility consists in using extensions of $Z-R$ relationships that include microphysical information and are more stable with scale. As shown in section 6, extensions of $Z-R$ relationships that involve DSD normalization parameters (like equation (34)) are more stable across scales. However, these modified relationships require the knowledge of the variability of $N_{0}^{*}$ and or $D_{m}$ to be applied. This could be investigated with algorithms like ZPHI that also 
work on microphysics. For other practical applications, solution I) seems more feasible in absence of microphysical information.

[109] III. Another solution to the scale-dependent biases can be considered. From the theoretical development in section 3, the dependence of $Z-R$ prefactors on scale is impacted by the degree of nonlinearity of the $Z-R$ relationships. More generally, a relationship $Y=\alpha X^{\beta}$ between two multifractal fields leads to a law of the form $\alpha(l) \propto l^{K(\beta)}$, where the exponent $K(b)$ will be close to 0 when $b$ is close to one. This means that relationships that are closer to proportionality are expectedly much more robust to scale dependence. In particular (as noted in section 3), the relationships used for rainfall estimation by polarimetric radars, i.e., $R=c K_{d p}^{d}$, are often less nonlinear than conventional $Z-R$ laws and should be more robust over many scales.

[110] An important additional remark is that the scale dependency demonstrated in section 3 is a purely statistical property. The empirical methodology followed in this paper was designed in order to eliminate some artefacts such as the influence of observational errors (low for the DBS instrument) or methodological ones (resolved by the systematic use of several regression methodologies). In real radar applications, these factors (and others) also impact $Z-R$ relationships and possibly differently across scales. The contributions of each source of errors need to be quantified and compared in order to reduce possible biases and error bars in radar-rainfall estimation.

\section{Summary}

[111] This paper has been structured by two main ideas. On the theoretical side, with the help of previous ideas [Tessier et al., 1993; Lovejoy et al., 2008], it has been shown that the extreme variability of rainfall processes, as described empirically by scaling and multifractal statistics, could lead to a scale dependency of the parameters in usual $Z-R$ power-law relationships used in quantitative radar-rainfall estimation. In particular, it has been demonstrated that if both $Z$ and $R$ follow conservative multifractal statistics, the exponent $b$ of $Z-R$ laws should be constant with scale whereas the prefactor $a$ should increase as a power law of scale (for usual values $b>1$ ), with moderate scaling exponent (of the order of 0.1 ).

[112] Empirically, the general qualitative behavior of the prefactors expected from theory is retrieved from the study of DBS data at small time scales (from $<1 \mathrm{~min}$ to $\sim 1 \mathrm{~h}$ ). Yet, we noticed a transition at 4 min time scale and only the larger scales seem to be theoretically interpretable. For nonlinear regressions and for regressions on conditional expected values (i.e., bin averages), the exponent $b$ does not vary much over the considered range of scales. For most regression methodologies, the prefactor $a$ of $Z-R$ laws is indeed found to follow approximate 0.1 to 0.15 power laws of integration times $\tau$, e.g., $a(\tau) \propto \tau^{0.1}$ that are rather coherent with theoretical considerations.

[113] While the scaling law of the prefactor $a$ has empirically been shown for the use of different integration times using a ground-based disdrometer, it seems likely that such a law remains valid for small spatial scales that are in the scope of weather radars (e.g., 1-10 km) since the hypotheses constraining the scale behavior of $Z-R$ laws (nonlinearity, multifractality) are still valid at such spatial scales. The existence of such a spatial property has been empirically supported by the works of Jaffrain and Berne [2012]. Clearly, it could impact radar-rainfall estimation at various levels: biases associated with radar pixel deformation with distance, inversion of spaceborne radar measurements using ground-based radar data, estimation of $Z-R$ relations at radar pixel scale using pointwise DSD information... The correction of such errors could be performed either by the means of the use of a supplementary scaling factor estimated from resolution and from statistical scaling parameters or by the use of alternative laws (e.g., relationships involving normalization parameters of the DSD or polarimetric $R-K_{\mathrm{dp}}$ laws).

[114] Further work is needed to systematically investigate the scale unrobustness of $Z-R$ relationships at (sub)mesoscale and especially in the spatial domain, with the help of different instruments (including radars). This would help to quantify the respective contributions of all factors impacting $Z-R$ parameters (statistical factors considered here vs. observational and other methodological errors) and to define the best strategy to minimize such errors. In particular, we expect that in the case of intense convective events, the scaling bias in $Z-R$ relationships could be important since the underlying variability of rainfall is higher. For such events, the errors in rainfall estimation could be very significant, with greater relative errors than those discussed in section 7.

[115] Let us finally highlight that the approach followed in the theoretical part of the paper seems extendable to the study of other fields that follow multifractal statistics over a given range of scales. As mentioned above, a relationship $Y=\alpha X^{\beta}$ between two (conservative) multifractal fields $X$ and $Y$ necessarily constraints the parameters $\alpha$ and $\beta$ such that $\beta$ is scale invariant and $\alpha(l) \propto l^{K(\beta)}$ where $l$ is the spatial (or temporal) scale. This could enlarge the implications of the present study to other rainfall fields (other DSD moments than $Z$ and $R$...) or possibly to other geophysical fields. This emphasizes the necessity to estimate geophysical parameters consistently with the resolution.

[116] Acknowledgments. S. Verrier acknowledges the Centre National d'Etudes Spatiales (CNES) for financial support.

\section{References}

Battan, L. J. (1973), Radar Observations of the Atmosphere, 324 pp., Univ. of Chicago Press, Chicago, USA.

Berne, A., and R. Uijlenhoet (2005), A stochastic model of range profiles of raindrop size distributions: Application to radar attenuation correction, Geophys. Res. Lett., 32, L10803, doi:10.1029/2004GL021899

Berne, A., J. Jaffrain, and M. Schleiss (2012), Scaling analysis of the variability of the rain drop size distribution at small scale, Adv. Water Resour., 45(9), 2-12, doi:10.1016/j.advwatres.2011.12.016, 2012.

Campos, E., and I. Zawadzki (2000), Instrumental uncertainties in Z-R relations, J. Appl. Meteorol., 39, 1088-1102.

Chapon, B., G. Delrieu, M. Gosset, and B. Boudevillain (2007), Variability of rain drop size distribution and its effect on the $Z-R$ relationship: A case study for intense Mediterranean rainfall, Atmos. Res., 87, 52-65, doi:10.1016/j.atmosres.2007.07.003.

Ciach, G. J., and W. F. Krajewski (1999), Radar-rain gauge comparisons under observational uncertainties, J. Appl. Meteorol., 38, 1519-1525.

Corrsin, S. (1951), On the spectrum of isotropic temperature fluctuations in an isotropic turbulence, J. Appl. Phys., 22, 469-473.

Delahaye, J.-Y., L. Barthès, P. Golé, J. Lavergnat, and J. P. Vinson (2006), A dual-beam spectropluviometer concept, J. Hydrol., 328, 110-120.

Dubrulle, B. (1994), Intermittency in fully developed turbulence: Log-Poisson statistics and generalized scale covariance, Phys. Rev. Lett., 73, 959-962.

Fabry, F. (1996), On the determination of scale ranges for precipitation fields, J. Geophys. Res., 101(D8), 12,819-12,826.

Fraedrich, K., and C. Larnder (1993), Scaling regimes of composite rainfall time series, Tellus, 45, 289-298.

Gires, A., I. Tchiguirinskaia, D. Schertzer, and S. Lovejoy (2012), Influence of the zero-rainfall on the assessment of the multifractal parameters, $A d v$. Wat. Resour., 45, 13-25.

Illingworth, A. J., and T. M. Blackman (2002), The need to represent raindrop size spectra as normalized gamma distributions for the interpretation of polarization radar observations, J. Appl. Meteorol., 41, 286-297. 


\section{VERRIER ET AL.: SCALE DEPENDENCY OF Z-R RELATIONSHIPS}

Jaffrain, J., and A. Berne (2012), Influence of the subgrid variability of the raindrop size distribution on radar rainfall estimators, J. Appl. Meteorol. Climatol., 51, 780-785.

Joss, J., and A. Waldvogel (1967), Ein Spektrograph für Niederschlagstropfen mit automatischer Auswertung, Pure Appl. Geophys., 68, 240-246.

Kolmogorov, A. N. (1941), Local structure of turbulence in an incompressible liquid for very large Reynolds numbers, Proc. Acad. Sci. URSS Geochem. Sect., 30, 299-303.

Lee, G. W., and I. Zawadzki (2005), Variability of drop size distribution: Time-scale dependence of the variability and its effects on rain estimation, J. Appl. Meteorol., 44, 241-255.

Lee, G., I. Zawadzki, W. Szyrmer, D. Sempere Torres, and R. Uijlenhoet (2004), A general approach to double-moment normalization of drop size distributions, J. Appl. Meteorol., 43(2), 264-281.

Levenberg, K. (1944), A method for the solution of certain problems in least squares, Ouart. Appl. Math., 2, p. 164-168.

de Lima, M. I. P., and J. Grasman (1999), Multifractal analysis of 15-min and daily rainfall from a semi-arid region in Portugal, J. Hydrol., 220, 1-11.

Lovejoy, S., and D. Schertzer (1995), Multifractals and rain, in New Uncertainty Concepts in Hydrology and Hydrological Modelling, edited by A. W. Kundzewicz, pp. 62-103, Cambridge Univ. press, Cambridge, UK.

Lovejoy, S., and D. Schertzer (2007), Scale, scaling and multifractals in geophysics: Twenty years on, in Nonlinear Dynamics in Geosciences, edited by A. Tsonis and J. Ellsner, pp. 311-337, Springer, New York, USA, doi:10.1007/978-0-387-34918-3 18.

Lovejoy, S., and D. Schertzer (2008), Turbulence, rain drops and the 11/2 number density law, New J. Phys., 10, 075,017, doi:10.1088/1367-2630/ 10/7/075017.

Lovejoy, S., and D. Schertzer (2010), Towards a new synthesis for atmospheric dynamics: Space-time cascades, Atmos. Res., 96, 1-52.

Lovejoy, S., and D. Schertzer (2013), The Weather and Climate: Emergent Laws and Multifractal Cascades, 496 pp., Cambridge Univ. Press, Cambridge, UK.

Lovejoy, S., D. Schertzer, and V. Allaire (2008), The remarkable wide range scaling of TRMM precipitation, Atmos. Res., 90(1), 10-32, doi:10.1016/j. atmosres.2008.02.016.

Lovejoy, S., J. Pinel, and D. Schertzer (2012), The global space-time cascade structure of precipitation: Satellites, gridded gauges and reanalyses, $A d v$. Water Resour., 45, 37-50, doi:10.1016/j.advwatres.2012.03.024.

Marquardt, D. (1963), An algorithm for least-squares estimation of nonlinear parameters, SIAM J, Appl. Math, 11, 431-44.

de Montera, L., L. Barthès, C. Mallet, and P. Golé (2009), The effect of rainno rain intermittency on the estimation of the universal multifractals mode parameters, J. Hydrometeorol., 10, 493-506.

Morin, E., W. F. Krajewski, D. C. Goodrich, X. Gao, and S. Sorooshian (2003), Estimating rainfall intensities from weather radar data: The scale-dependency problem, J. Hydrometeorol., 4, 782-797.

Novikov, E. A., and R. Stewart (1964), Intermittency of turbulence and spectrum of fluctuations in energy-disspation, Izv. Akad. Nauk. SSSR. Ser. Geofiz., 3, $408-412$

Obukhov, A. M. (1949), Structure of the temperature field in a turbulent flow, Izv. Akad. Nauk S.S.S.R, Ser. Geograf. Geofiz., 13, 58-69.

Pathirana, A., S. Herath, and T. Yamada (2003), Estimating rainfall distributions at high temporal resolutions using a multifractal model, Hydrol. Earth Syst. Sci., 7, 668-679.
Rosenfeld, D., and C. W. Ulbrich (2003), Cloud microphysical properties, processes, and rainfall estimation opportunities, Meteorol. Monogr., 30, 237.

Rysman, J. F., S. Verrier, Y. Lemaître, and E. Moreau (2013), Space time variability of the rainfall over the western Mediterranean region: A statistical analysis, submitted to J. Geophys. Res. Atmos., (in revision).

Sachidananda, M., and D. S. Zrnic (1987), Rain rate estimates from differential polarization measurements, J. Atmos. Oceanic Technol., 4, 588-598.

Schertzer, D., and S. Lovejoy (1987), Physically based rain and cloud modeling by anisotropic, multiplicative turbulent cascades, J. Geophys. Res., 92(D8), 9692-9714.

Schertzer, D., and S. Lovejoy (1997), Universal multifractals do exist!, J. Appl. Meteorol., 36, 1296-1303.

Schertzer, D., S. Lovejoy, and P. Hubert (2002), An introduction to stochastic multifractal fields, in Mathematical Problems in Environmental Science and Engineering, Ser. in Contemporary Appl. Math., vol. 4, edited by A. Ern, and L. Weiping, pp. 106-179, Higher Educ. Press, Beijing.

Sekhon, R. S., and R. C. Srivastava (1970), Snow size spectra and radar reflectivity, J. Atmos. Sci., 27, 299-307.

Sempere Torres, D., J. M. Porra, and J. D. Creutin (1994), A general formulation for raindrop size distribution, J. Appl. Meteorol., 33(12), 1494-1502.

Sempere Torres, D., J. M. Porra, and J. D. Creutin (1998), Experimenta evidence of a general description for raindrop size distribution properties, J. Geophys. Res., 103(D2), 1785-1797.

She, Z. S., and E. Levêque (1994), Universal scaling laws in fully developed turbulence, Phys. Rev. Lett., 72, 336-339.

Smith, J. A., and W. F. Krajewski (1993), A modeling study of rainfall ratereflectivity relationships, Water Resour. Res., 29(8), 2505-2514.

Steiner, M., and J. A. Smith (2004), Scale dependence of radar-rainfall ratesAn assessment based on raindrop spectra, J. Hydrometorol., 5, 1171-1180.

Tessier, Y., S. Lovejoy, and D. Schertzer (1993), Universal multifractals in rain and clouds: Theory and observations, J. Appl. Meteorol., 32, 223-250.

Testud, J., E. Le Bouar, E. Obligis, and M. Ali-Mehenni (2000), The rain profiling algorithm applied to polarimetric weather radar, J. Atmos. Oceanic Technol., 17, 332-356.

Testud, J., S. Oury, R. A. Black, P. Amayenc, and X. K. Dou (2001), The concept of "normalized" distribution to describe raindrop spectra: A tool for cloud physics and cloud remote sensing, J. Appl. Meteorol., 40(6), 1118-1140.

Uijlenhoet, R. (2001), Raindrop size distributions and radar reflectivity-rain rate relationships for radar hydrology, Hydrol. Earth Syst. Sci., 5(4), 615-627.

Uijlenhoet, R., M. Steiner, and J. A. Smith (2003), Variability or raindrop size distribution in a squall line and implication for radar rainfall estimation, J. Hydrometeorol., 4, 43-61.

Verrier, S., L. de Montera, L. Barthès, and C. Mallet (2010), Multifractal analysis of African monsoon rain fields, taking into account the zero rain-rate problem, J. Hydrol., 389, 111-120.

Verrier, S., C. Mallet, and L. Barthès (2011), Multiscaling properties of rain in the time domain, taking into account rain support biases, J. Geophys Res., 116, D20119, doi:10.1029/2011JD015719.

Yaglom, A. M. (1966), The influence of the fluctuation in energy dissipation on the shape of turbulent characteristics in the inertial interval, Sov. Phys. Dokl., 2, 26-30.

Yu, N. (2012), Précipitations méditerranéennes intenses - Characterisation microphysique et dynamique dans l'atmosphère et impacts au sol, thèse Université Joseph Fourier, Grenoble, France, Laboratoire d'Etude des Transferts en Hydrologie et Environnement, Grenoble, France. 